\title{
Spacecraft Trajectory Optimization: A review of Models, Objectives, Approaches and Solutions
}

\author{
Abolfazl Shirazi ${ }^{1}$ \\ Basque Center for Applied Mathematics - BCAM, Bilbao, 48009, Spain \\ Josu Ceberio ${ }^{2}$ \\ University of the Basque Country UPV/EHU, Donostia, 20018, Spain \\ Jose A. Lozano ${ }^{3}$ \\ Basque Center for Applied Mathematics - BCAM, Bilbao, 48009, Spain \\ University of the Basque Country UPV/EHU, Donostia, 20018, Spain
}

\begin{abstract}
This article is a survey paper on solving spacecraft trajectory optimization problems. The solving process is decomposed into four key steps of mathematical modeling of the problem, defining the objective functions, development of an approach and obtaining the solution of the problem. Several subcategories for each step have been identified and described. Subsequently, important classifications and their characteristics have been discussed for solving the problems. Finally, a discussion on how to choose an element of each step for a given problem is provided.
\end{abstract}

Keywords: Trajectory, Mathematical model, Objective, Approach, Solution, Optimization, Algorithm, Metaheuristics

\footnotetext{
${ }^{1}$ Ph.D Fellow, Machine Learning Group, ashirazi@bcamath.org

${ }^{2}$ Lecturer, Intelligent Systems Group, josu.ceberio@ehu.eus

${ }^{3}$ Professor, ja.lozano@ehu.eus
} 


\section{Introduction}

The spacecraft trajectory optimization problem can be described as the discovery of a trajectory that satisfies some criteria, including initial and terminal conditions. In recent years, considerable progress has been made in the development of methods to find optimal trajectories for spacecraft in various space missions. Within this progress, each step in spacecraft trajectory design can be categorized according to the elements that are involved in finding a solution to the optimal trajectory problem, such as the mathematical model, objective, approach, or, more importantly, the method, technique and algorithm.

Perhaps the first serious attempt to categorize methods for spacecraft trajectory optimization was made by Betts [1] in 1998. The main classification made by Betts considered two famous methods, known as direct and indirect methods, and the primary related techniques in each were summarized. In 2012, Conway [2] made another comprehensive contribution to the numerical approaches applied in dynamical systems. He provided an excellent overview of different methods, similar to Betts' survey, along with practical examples. However, the dynamical systems considered in his survey are in general form. Other attempts are also made but limited to specific space missions, such as Earth-Moon trajectories [3], space rendezvous [4], planetary entry [5] and libration points [6], [7]. Different classifications are presented for spacecraft trajectory optimization problems in these researches. Based on the purposes of their taxonomy, each approach or solution has its own advantages and disadvantages [8]. These surveys focused on specific steps of the whole process rather than a general scheme for spacecraft trajectory optimization. Moreover, it is clear from the literature that comparing different taxonomies can be time consuming, although it is generally less complicated than developing

one from scratch. While a great deal of research has been done regarding the methods and techniques, an outline that categorizes the key elements within the general process of spacecraft trajectory optimization is missing. This paper presents such a scheme and it is considered complementary to all of the previously published survey articles in this subject. It reflects the research that has been done over the past decade while simultaneously providing a road map for the general process of spacecraft trajectory optimization.

Before proceeding to the details of the review, it is important to distinguish between several terms in spacecraft trajectory optimization terminology. By looking through the literature, it can be highlighted that a vast 
number of papers are dedicated to spacecraft trajectory optimization with different terminologies. Approaches, solutions, methods, strategies, techniques and other terms are often used interchangeably. This review also tries to make a distinction between such terms, and uses a clear terminology in order to avoid misunderstandings and confusion when referring to a method, approach, technique or algorithm. It should also be noted that sometimes different parameters are shown with same symbols in literature. Therefore, in this review, every newly introduced parameter in the equations is defined locally in order to avoid misunderstandings with other possible parameters with same symbols.

The entire process of solving a spacecraft trajectory optimization problem can be divided in four steps as depicted in Fig. 1. This general process includes mathematical modeling of system dynamics, defining appropriate objectives, developing an approach and, lastly, achieving the solution. Traces of these key elements can be found in textbooks by Betts [9] and Conway $[10]$.

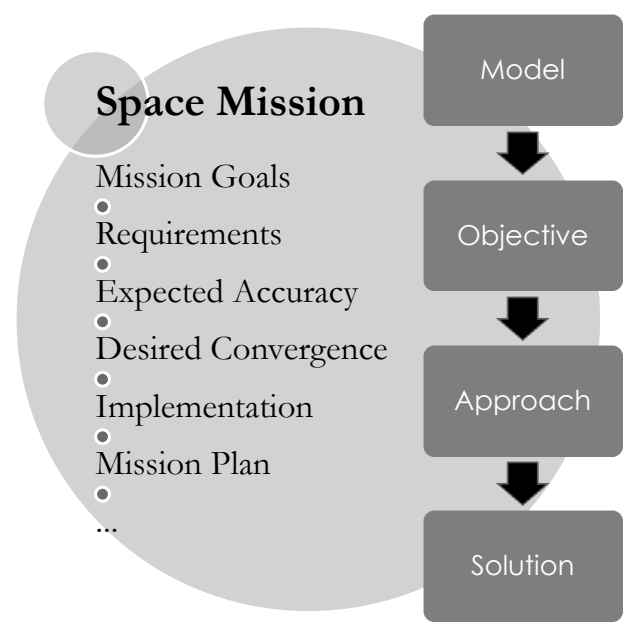

Figure 1: General scheme of spacecraft trajectory optimization process

These steps are represented by model, objective, approach and solution respectively. On the other hand, each space mission has several components such as mission requirements, goals, expected accuracy, desired convergence, mission plan, etc. Each of these factors affects the steps of the mentioned process differently. Therefore, it is important to focus on the taxonomies in each step based on the space mission components in order to make a good 
decision when choosing a model or employing a method in the spacecraft trajectory optimization process.

The first step to solve not just a spacecraft trajectory optimization problem but indeed any orbital mechanics problem involves a firm understanding of the dynamics inherent in the system. It refers to the mathematical modeling of the problem which involves choosing a set of states to represent the system and derivation of motion equations for spacecraft.

The second step is handling the mission objectives via defining cost functions. Two categories can be considered for this step, one according to the type of the objectives and the other according to the number of objectives.

As the third step, the type of methods and techniques which are dedicated to solving the trajectory design problem are the main feature that characterizes the approach. This step is divided into two categories called analytical and numerical approaches. Analytical approaches are mainly based on the well-known optimal control theory [11]. The purpose of this theory is the determination of a time history of controls that satisfies the physical constraints of the system while minimizing some performance criteria [12] i.e., the cost functions defined in the previous step. There also exist several numerical approaches to solve optimization problems related to space transfers [10]. They fall essentially into two main categories. The first one is called direct methods, which attempt to find the minimum of the cost function by considering state and input vectors. The second one is indirect methods, which involve adjoint equations alongside state and input vectors based on the Pontryagin's Principle [11]. Each one of these two large categories is characterized by both positive and negative aspects, intrinsically limiting their operational fields.

The fourth step is to solve the problem regarding the developed approach. If the analytical approach is developed in the third step, the solution is likely to be a closed form analytical solution. However, if the numerical approach is used, the problem usually turns into a black-box optimization problem and needs numerical algorithms to achieve a solution. Most of the spacecraft trajectory optimization problems end in the latter form, to be solved by numerical techniques rather than by means of an analytical solution. The reason is that a typical spacecraft optimization problem does not have a closed form solution due to its nonlinearity, unless specific conditions and assumptions are considered in the approach. Such assumptions may limit the matching between simulation and reality in spacecraft motion.

This review tries to propose a complete taxonomy of spacecraft trajec- 
tory optimization problems, along with recently developed concepts and traditional approaches, which covers most of the aspects of this field. The idea is to bring the advantages and disadvantages of various models, objectives, approaches and solutions based on the findings of more than two hundred research papers. In contrast, this review excludes many of the technical details and, instead, provides a road map of currently available tools. General concepts are briefly described, and references are included for further investigation. In addition, this paper tries to consolidate seemingly different concepts, methods, and terminology stemming from diverse applications. While a great deal of spacecraft trajectory optimization research has been carried out in the aerospace community, this review attempts to draw from work that has been done in other disciplines as well. It also provides conclusions that can be useful for other disciplines such as applied mathematics and engineering.

This review is organized as follows: The next four sections are dedicated to the taxonomies of the four steps mentioned in the process of spacecraft trajectory optimization respectively. Section 2 provides the mathematical models required in order to formulate the necessary components of the spacecraft trajectory optimization problem. It outlines several choices of mathematical sets and their corresponding equations of motion according to different categories of space missions. Objective functions in spacecraft trajectory optimization problems, their representation and types are discussed in Section 3. Section 4 details the approaches used in solving the spacecraft trajectory optimization problem, as well as comparisons of different methods and techniques. Section 5 is dedicated to optimization algorithms, including nonlinear programming and metaheuristics. This section aims to taxonomize the optimization algorithms according to space missions and their usage in spacecraft trajectory optimization problems. Section 6 summarizes the discussions from this review. It also presents suggestions for future study and new trends in trajectory optimization of spacecraft. Finally, the conclusions are provided in Section 7.

\section{Model}

As the first step of facing the spacecraft trajectory optimization problem, the dynamics of the spacecraft need to be mathematically modeled. The spacecraft trajectory model can be referred to a set of ordinary differential

equations representing a path or time history of position and velocity of the 
spacecraft. The equations of motion for the spacecraft which serves as the model can be generally described in first order form as follows [12]:

$$
\overrightarrow{\dot{x}}=f(\vec{x}(t), \vec{u}(t), t)
$$

where $t$ represents the time, $\vec{x}(t)$ is an $n$-dimensional time history of the state vector and $\vec{u}(t)$ is an $m$-dimensional time history of the control vector, which serves as the system input. The state vector contains the state variables which can be the position and the velocity vectors of the spacecraft. This general representation is used in the literature as the basic mathematical model for spacecraft trajectory and can be categorized in different aspects and forms, as depicted in Fig. 2.

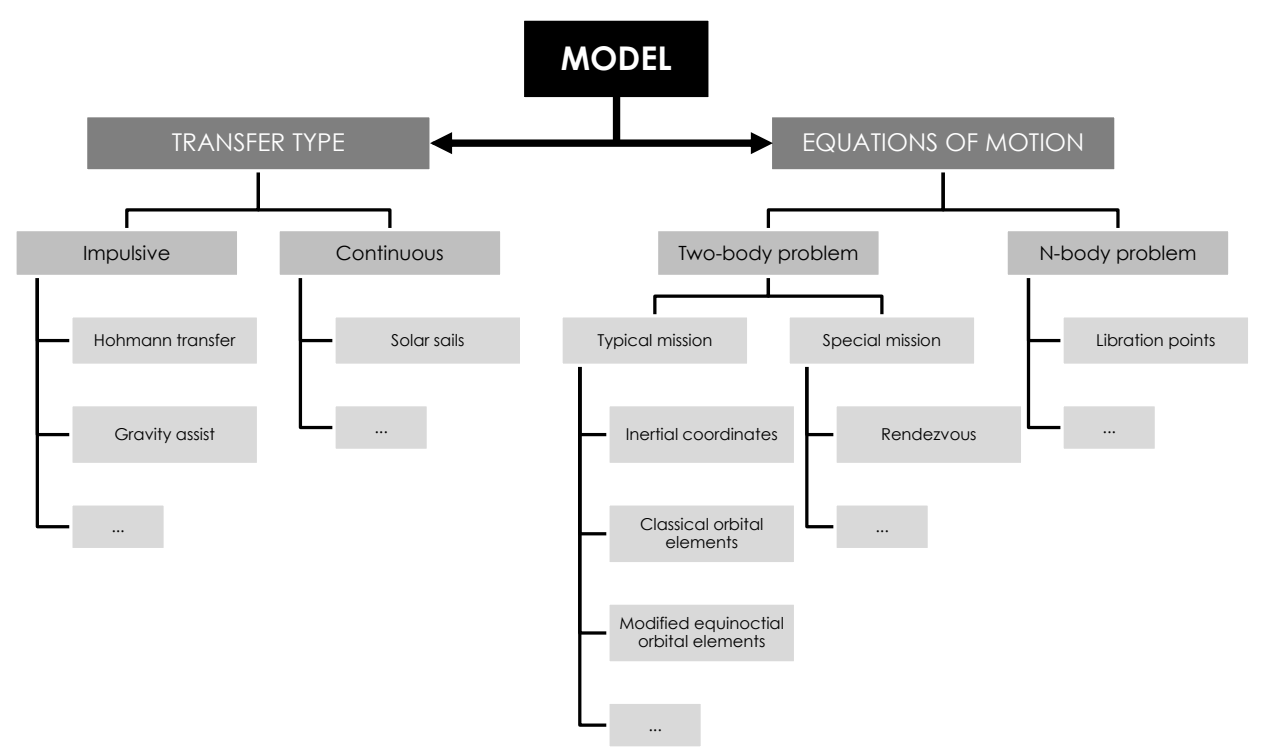

Figure 2: Taxonomy of mathematical models in spacecraft trajectory optimization

The overall taxonomy of mathematical models of the spacecraft in trajectory optimization problems consists of two minor categories which are transfer type and equations of motion. While the behavior of the input $\vec{u}(t)$ is the matter of interest in the first category, the focus in the second category is on the representation of the whole differential equations as in $f(\vec{x}(t), \vec{u}(t), t)$. Although other aspects, such as dimensions (2D and $3 \mathrm{D})$, could be also considered in the taxonomy, these two categories are chosen in this taxonomy since they can cover and classify most of the research according to the liter- 
ature.

\subsection{Models based on transfer type}

In mathematical modeling of the spacecraft trajectory in an orbit transfer, the simulation of the system inputs is an important issue which has a great effect on the trajectory optimization process. Depending on the type of space mission, the model can be either impulsive or continuous.

\subsubsection{Impulsive model}

Mathematical modeling based on impulsive model is the traditional procedure used to simulate the spacecraft maneuver. In this modeling, the inputs of the system are assumed to be zero $\vec{u}(t)=0$ and the maneuver by the spacecraft is considered as sudden velocity increments $(\Delta v>0)$ with zero burn times $(\Delta t=0)$. If the gravitational acceleration of only one giant mass (for example the Earth) is considered for the problem, the presented differential equation of motion based on state variables in Eq. 1 may be reduced and simplifies into some algebraic equations based on orbital elements. This model is relatively simple to be used in simulation of space trajectory with large accelerations and a rapid spacecraft response to commanded maneuvers. It allows to simulate nearly instantaneous velocity changes necessary for large orbital maneuvers [13]. Impulsive model is typically used when engines with relatively low specific impulse $\left(I_{s p}\right)$ and high thrust level are employed. This kind of model, better known as the Kepler model, which was first proposed by Sims and Flanagan [14] to approximate low-thrust trajectories as a series of impulsive $\Delta v$ 's connected by conic arcs, is depicted in Fig. 3.

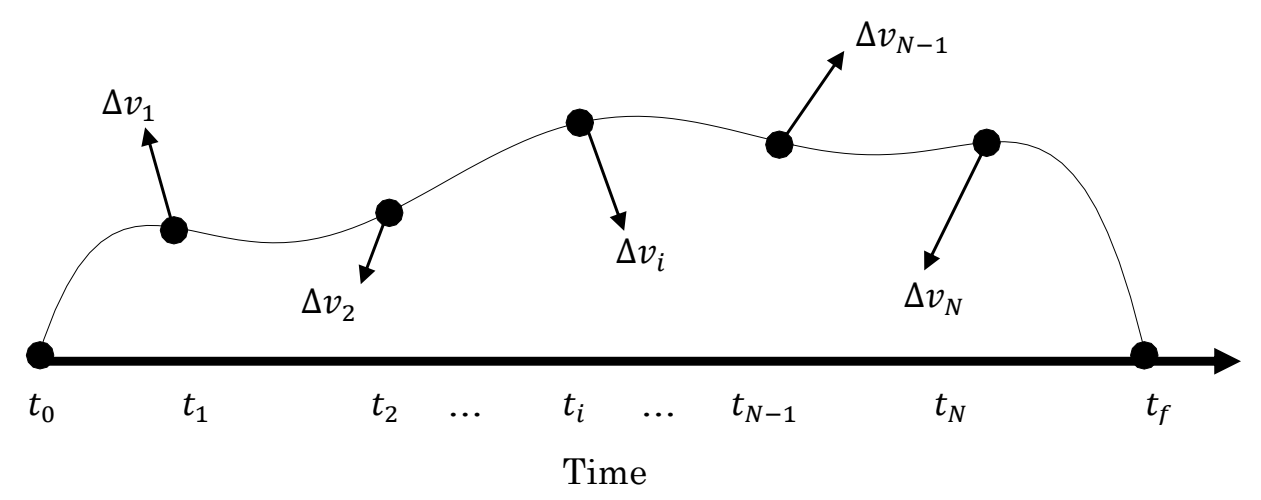

Figure 3: Impulsive discretization scheme 
In the impulsive model, a segment $\left(t_{i}\right.$ to $\left.t_{i+1}\right)$ corresponds to an impulsive $\Delta v$ which can be analyzed by an analytical Kepler propagation with respect to a primary body (Sun, Earth or other planets). Since a closed-form solution is usually known for the state propagation, no numerical integration of the equations of motion is needed, which results in fast computations. The wellknown Hohmann transfer [13] is the most practical transfer in which the impulsive model is taken into account for initial design and analysis of orbits in space mission [15].

An advanced concept of impulsive model is called impulsive thrusting. In this model, the trajectory is locally treated as continuous when the engine is on, and the thrust level and the burn time will be considered in the problem. This type of model is sometimes used for optimization of continuous thrust orbit transfers. One example is [16], in which a multi-impulse extended method is proposed for low-thrust trajectory optimization. Some applications of this model in the literature are shown in Table 1, which are tabulated based on the type of space mission.

\begin{tabular}{cc}
\hline \hline Mission & References \\
\hline Rendezvous & {$[17][18][19][20][21]$} \\
\cline { 2 - 2 } Typical transfers & {$[22][23][24][25][26][16][27]$} \\
\cline { 2 - 2 } Gravity assist & {$[24][25][28][29][30][31]$} \\
& {$[32][33][34][35][36]$} \\
\hline \hline
\end{tabular}

Table 1: Spacecraft trajectory design and optimization based on impulsive model $\backslash$ thrust

As stated, the impulsive model is suitable for space missions with sudden velocity increment. Thrust phases for these missions are typically short compared to the overall mission time, which makes the problem relatively straightforward. As a result, thrust arcs are modelled as isolated, singular events, and the continuous problem can be reduced to a discrete optimization problem which can be represented with the impulsive model. In such cases, the impulsive $\Delta v$ 's readily represents deep space maneuvers, especially for space missions with several segments (such as gravity assist maneuvers). If no maneuver is needed at the beginning of a segment, the optimizer simply drives the corresponding $\Delta v$ magnitude to zero. The optimization of the number of impulses, as well as their respective locations, is therefore automatically tackled. 


\subsubsection{Continuous model}

The second type for mathematical modeling of the spacecraft is the continuous model. Mathematical models based on this concept are more precise but also more complicated in comparison to impulsive models, since the trajectory is dealt with considering non-zero inputs $(\vec{u}(t) \neq 0)$. The comparison between impulsive and continuous models is implicitly a mirror of comparing high and low-thrust space missions. From the viewpoint of performance, low-thrust propulsion can improve fuel consumption efficiency for space missions due to their extremely high $I_{s p}$ compared with high-thrust chemical propulsion. However, typical low-thrust trajectories present a major challenge namely the extremely low forces that they generate. Table 2 provides detailed characteristics for some specific low and high-thrust propulsion systems [37], [38], [39] [40], [41].

\begin{tabular}{ccc}
\hline \hline Propulsion system & Thrust $(\mathrm{N})$ & $I_{s p}(\mathrm{~s})$ \\
\hline Chemical engine & $0.1-10^{6}$ & $140-460$ \\
Cold gas thruster & $0.05-200$ & $50-250$ \\
Resisto-jet & $0.002-0.1$ & $150-8000$ \\
Arcjet & $0.002-0.7$ & $400-1500$ \\
Ion thruster & $1 \times 10^{-5}-0.2$ & $1500-5000$ \\
Hall thruster & $1 \times 10^{-5}-1$ & $1500-6000$ \\
Pulsed plasma,thruster & $5 \times 10^{-5}-0.01$ & $500-2000$ \\
Solar sail & $0.001-0.1$ & $\infty$ \\
\hline \hline
\end{tabular}

Table 2: Characteristics of typical propulsion systems

Unlike high-thrust trajectories, the transfer time in low-thrust trajectories is relatively high. Therefore, the continuous model is more adequate for lowthrust trajectories. However, there is some research which deals with the employment of continuous model in analyzing high-thrust transfers as well. For example, in [42], the problem of the optimal space trajectory for the mission to the Apophis asteroid approaching the Earth has been studied with the employment of a continuous model for the departure phase from Earth.

The general representation of continuous transfer is the extension of the 
Newton equation for the N-body problem as in Eq. 2.

$$
\vec{r}=-G \sum_{i=1}^{n} \frac{m_{i}\left(\vec{r}-\vec{r}_{i}\right)}{\left|\vec{r}-\vec{r}_{i}\right|^{3}}+\vec{\Gamma}
$$

where $\vec{r}$ is the position of the spacecraft, $\overrightarrow{r_{i}}$ are the positions of $n$ celestial bodies with masses $m_{i}$, and $G$ is the gravitational constant. $\vec{\Gamma}$ represents the summation of any accelerations due to sources other than the gravitational force of celestial bodies, such as the space perturbations or the thrust provided by the spacecraft propulsion system [43]. It is clear that, by considering the position vector $\vec{r}$ and its time derivative $\overrightarrow{\dot{r}}$ as the state vectors (i.e., $\vec{x}(t)=\left[\begin{array}{ll}\vec{r} & \vec{r}\end{array}\right]$ ), Eq. 2 will be a specific form of the general model representation as in Eq. 1 while considering $\vec{\Gamma}$ as a function of $\vec{u}(t)$. This is the general equation for any continuous spacecraft trajectory optimization problem. Obviously, by setting $\vec{u}(t)=0$ and considering the maneuvers as sudden velocity increments, the continuous model will turn into the impulsive model. For specific missions and applications such as unperturbed orbits around Earth, the orbit propagation may be simplified to orbital elements. Therefore, the complexity of the model can be changed for various applications, from very simplistic (for example Hohmann transfer) to heavily complicated (ex. low-thrust interplanetary transfer).

\subsection{Models based on equations of motion}

Besides the concept of a mathematical model for spacecraft trajectory optimization, the representation of the dynamics of the spacecraft motion is a key to categorize the model. Different forms of Eq. 2 are considered in the literature depending on the space mission, falling into two main groups of two-body problems and N-body problems. Obviously, this category does not conflict with the previous one. In the other words, one can simulate two-body problems or N-body problems with either impulsive or continuous models.

\subsubsection{Typical two-body problems}

The simplest model for spacecraft dynamics is the two-body problem model [13]. This model begins with two point masses and describes their mutual gravitational attraction to each other [15]. In this modeling, the mass of the spacecraft is assumed to be much smaller than the mass of the body it is orbiting. This allows the spacecraft's mass and its gravitational 
effects on the larger body to be neglected. Moreover, the frame of reference is inertial. This allows for derivatives to be taken without regarding the motion of the reference frame. Besides, both the celestial body and the spacecraft are supposed to be point masses and no other forces are applied to either body [44]. These assumptions allow for the basic formulation of the two-body problem, however they constitute an imperfect model.

\section{Inertial coordinates}

The most common mathematical model of spacecraft dynamics regarding the mentioned assumptions for typical two-body problems can be described as the well-known non-Keplerian two-body problem equation [13], [44]:

$$
\vec{r}=-\frac{\mu}{r^{3}} \vec{r}+\vec{\gamma}
$$

This equation of motion is best described initially using an independent inertial coordinate frame. In this equation, $\vec{r}$ denotes the position of the spacecraft relative to inertial coordinate system, $\mu$ is the gravitational constant of the central mass, and $\vec{\gamma}$ is the acceleration due to engine thrust. Rewriting this equation in scalar form yields the following set of first-order derivatives [15]:

$$
\left(\begin{array}{c}
\dot{r}_{x} \\
\dot{r}_{y} \\
\dot{r}_{z} \\
\dot{v}_{x} \\
\dot{v}_{y} \\
\dot{v}_{z}
\end{array}\right)=\left(\begin{array}{c}
v_{x} \\
v_{y} \\
v_{z} \\
-\frac{\mu}{r^{3}} r_{x}+\gamma_{x} \\
-\frac{\mu}{r^{3}} r_{y}+\gamma_{y} \\
-\frac{\mu}{r^{3}} r_{z}+\gamma_{z}
\end{array}\right)
$$

where $r_{x}, r_{y}, r_{z}$ are the position components $\left(r=r_{x} \vec{i}+r_{y} \vec{j}+r_{z} \vec{k}\right), v_{x}, v_{y}, v_{z}$ are the velocity components $\left(\vec{r}=v_{x} \vec{i}+v_{y} \vec{j}+v_{z} \vec{k}\right)$ and $\gamma_{x}, \gamma_{y}, \gamma_{z}$ are the acceleration components $\left(\vec{\gamma}=\gamma_{x} \vec{i}+\gamma_{y} \vec{j}+\gamma_{z} \vec{k}\right)$ in Earth Centered Inertial (ECI) frame.

Besides Cartesian form, cylindrical coordinates are sometimes considered in research as follows.

$$
\left(\begin{array}{c}
\ddot{r}-r \dot{\theta}^{2}+\frac{\mu}{s^{3}} r \\
r \ddot{\theta}+2 \dot{r} \dot{\theta} \\
\ddot{z}+\frac{\mu}{s^{3}} r
\end{array}\right)=\left(\begin{array}{c}
\gamma_{r} \\
\gamma_{\theta} \\
\gamma_{z}
\end{array}\right)
$$


where $s=\sqrt{r^{2}+z^{2}}$, and $\gamma_{r}, \gamma_{\theta}, \gamma_{z}$ are the acceleration components in cylindrical coordinate systems. These general trajectory equations of motion are vastly used in many spacecraft trajectory optimization problems [45], specifically for analyzing perturbed orbits [46] and low-thrust transfers [47]. Although the Cartesian and cylindrical forms are often used for typical spacecraft trajectory optimization problems [48], other forms based on the variation of parameters are sometimes used in spacecraft trajectory optimization.

\section{Classical orbital elements}

Another form of mathematical model for spacecraft trajectory optimization is in terms of classical orbit elements. The six classical orbital elements [44] can be derived from the position and velocity vectors directly [13]. Nevertheless, sometimes the following Lagrange equations are used in mathematical modeling of spacecraft dynamics [44]:

$$
\begin{aligned}
\frac{d a}{d t}= & \left(\frac{2 e \sin \theta}{n \sqrt{1-e^{2}}}\right) \gamma_{r}+\left(\frac{2 a \sqrt{1-e^{2}}}{n r}\right) \gamma_{t} \\
\frac{d e}{d t}= & \left(\frac{\sqrt{1-e^{2}} \sin \theta}{n a}\right) \gamma_{r}+\left(\frac{\sqrt{1-e^{2}}}{n a^{2} e}\left(\frac{a^{2}\left(1-e^{2}\right)}{r}-r\right)\right) \gamma_{t} \\
\frac{d i}{d t}= & \left(\frac{r \cos (\omega+\theta)}{n a^{2} \sqrt{1-e^{2}}}\right) \gamma_{n} \\
\frac{d \Omega}{d t}= & \left(\frac{r \sin (\omega+\theta)}{n a^{2} \sqrt{1-e^{2}} \sin i}\right) \gamma_{n} \\
\frac{d \omega}{d t}= & \left(-\frac{\sqrt{1-e^{2}} \cos \theta}{n a e}\right) \gamma_{r}+\left(\frac{\sqrt{1-e^{2}}}{n a e}\left(1+\frac{r}{a\left(1-e^{2}\right)}\right) \sin \theta\right) \gamma_{t}- \\
& \left(\frac{r \cot i \sin (\omega+\theta)}{n a^{2} \sqrt{1-e^{2}}}\right) \gamma_{n} \\
\frac{d M}{d t}= & \left(\frac{\left(1-e^{2}\right) \cos \theta}{n a e}-\frac{2 r}{n a^{2}}\right) \gamma_{r}-\left(\frac{1-e^{2}}{n a e}\left(1+\frac{r}{a\left(1-e^{2}\right)}\right) \sin \theta\right) \gamma_{t}+ \\
& n
\end{aligned}
$$

where the classical orbital elements, $a, e, i, \Omega, \omega, M$ are semi-major axis, eccentricity, inclination, right ascension of ascending node (RAAN), argument of perigee and mean anomaly. $n$ is the mean motion, defined as $n=\sqrt{\frac{\mu}{a^{3}}}$ and $\theta$ is the true anomaly. The parameters $\gamma_{r}, \gamma_{t}, \gamma_{n}$ denote radial, tangential, and normal components, respectively [49]. 
The advantage of using this set of equations is that they provide the variation of classical orbital elements directly from the acceleration without the need for state variables. This method of defining an orbital state is intuitive but unfortunately has a number of singularities that tend to complicate the equations of motion. For instance, at zero inclination the right ascension of ascending node loses meaning. Similarly, for zero eccentricity the argument of perigee becomes indistinguishable from the true anomaly. These singularities can be clearly seen in their equations of motion. Due to the existence of these singularities, the classical orbital elements are not necessarily the best set of states for numerical analysis.

\section{Modified equinoctial orbital elements}

The other model of completely defining an orbit is by the use of the modified equinoctial orbital elements. This element set maintains the mathematical advantages of the classical orbital elements without going singular for circular or prograde equatorial orbits. The set of differential equations defining the spacecraft dynamics based on equinoctial orbital elements is as follows $[9]$ :

$$
\begin{aligned}
\frac{d p}{d t} & =2 \sqrt{\frac{p^{3}}{\mu}} \frac{1}{W} f_{N} \\
\frac{d f}{d t} & =\sqrt{\frac{p}{\mu}} \frac{1}{W}\left(W \sin (L) f_{s}+A(L) f_{N}-g(h \sin (L)-k \cos (L)) f_{W}\right) \\
\frac{d g}{d t} & =\sqrt{\frac{p}{\mu}} \frac{1}{W}\left(-W \cos (L) f_{s}+B(L) f_{N}+f(h \sin (L)-k \cos (L)) f_{W}\right) \\
\frac{d h}{d t} & =\frac{1}{2} \sqrt{\frac{p}{\mu}} \frac{X}{W} \cos (L) f_{W} \\
\frac{d k}{d t} & =\frac{1}{2} \sqrt{\frac{p}{\mu}} \frac{X}{W} \sin (L) f_{W} \\
\frac{d L}{d t} & =\sqrt{\frac{\mu}{p^{3}}} W^{2}+\sqrt{\frac{p}{\mu}} \frac{1}{W}(h \sin (L)-k \cos (L)) f_{W}
\end{aligned}
$$


where the following abbreviations have been used:

$$
\begin{aligned}
s & =\sqrt{1-f^{2}-g^{2}} \\
X & =1+h^{2}+k^{2} \\
W & =1+f \cos (L)+g \sin (L) \\
A(L) & =f+\cos (L)(1+W) \\
B(L) & =g+\sin (L)(1+W)
\end{aligned}
$$

where $p, f, g, h, k$ and $L$ are the modified equinoctial orbital elements. Also, $f_{N}, f_{S}$, and $f_{W}$ are components of the perturbing acceleration in the directions perpendicular to the radius vector in the direction of motion, along the outward radius vector, and normal to the orbital plane in the direction of the angular momentum vector, respectively. This set of equations is employed in a lot of research with various space missions including interplanetary transfers with gravity assist maneuvers [50], [51]. Besides, the use of modified equinoctial elements to describe the osculating orbits is a good choice due to the easy formulation and robustness to uncertainties. See [52] for an instance regarding these characteristics.

\section{Comparison}

Other forms of differential equations may be used as the model for typical two-body problems in spacecraft trajectory optimization. To be more specific, there are twenty two identified candidate orbit element sets plus variations defined in terms of Euler angles, Euler parameters, functions of classical elements, quaternions, set-III elements, fast or slow variables, or canonical variables. These other forms of orbital elements are well explained in a survey by Hintz [53]. However, the model presented by the equation of state vectors including position and velocity is used more frequently in spacecraft trajectory optimization problems. The three sets of equations for modeling the two-body problems are compared in Table 3.

\begin{tabular}{lccc}
\hline \hline & Inertial coordinates & $\begin{array}{c}\text { Classical orbital } \\
\text { elements }\end{array}$ & $\begin{array}{c}\text { Modified equinoctial } \\
\text { orbital elements }\end{array}$ \\
\hline Having physical meaning & Normal & High & Low \\
Extending to other forms & Easy & Hard & Hard \\
Suffering from singularities & No & Yes & No \\
Practicality for numerical averaging & No & No & Yes \\
Complexity of equations & Low & High & Medium \\
\hline \hline
\end{tabular}

Table 3: Comparison of dynamic models for continuous thrust transfers 
As it has been demonstrated, when external acceleration is introduced, time variation of classical orbital elements can be calculated based on the standard variation of physical parameters. Euler angles are used to parameterize the orientation of the orbit plane. However, due to the inherent singularities of the Euler angles, the variational equations may become singular for zero eccentricity and/or zero inclination because $\Omega$ and $\omega$ are indeterminate for $i=0$, or $\pi$ and $\omega$ is indeterminate for $e=0$.

While the modified equinoctial orbital elements avoid the singularities of the classical orbital elements, the main disadvantage of using them is that from direct inspection it is not intuitively obvious what is happening physically to the system. The classical orbital elements directly relate to the physical geometry of the orbit and are much simpler to directly interpret than the equinoctial orbital elements.

There is significant freedom in the choice of a suitable set of state variables or elements. The modified equinoctial element set is the only one that is nonsingular for all values of eccentricity and inclination. This set also employs elements that are not far from the classical ones, so that transforming and interpreting them in terms of physically significant parameters is relatively easier than using classical orbital elements. Therefore, it is advisable to use the modified equinoctial orbit element set for the research and technology development task. They can also be used for the integration of orbits with special and general perturbations, as well as differential corrections in orbit determination. However, the other orbit element sets could prove to be convenient in specific applications where the singularities are not a problem $[53]$.

\subsubsection{Rendezvous}

Besides the models for typical two-body problems, the general equation of motion can be reformulated and turned into new representations regarding any special space missions. One of the challenging space missions in literature is the space rendezvous. Rendezvous in space between two spacecraft is accomplished when both space vehicles attain the same position vector and velocity vector at the same time. However, at the time the rendezvous sequence is initiated, they may be very far apart, possibly with one satellite at liftoff. The first part of a rendezvous sequence is the phasing step, which is to perform the maneuvers in the timing sequence that will bring the two satellites into close proximity. For this step, the state vectors model which includes the position and velocity [44] is usually valid and selected as the 
general dynamic equation for describing the spacecraft motion.

The next step is the terminal rendezvous. It performs the maneuvers that induce the relative motion between the spacecraft that is required for rendezvous and docking, i.e., the motion of one spacecraft (chaser or active vehicle) with respect to the other (target or passive vehicle). The coordinate frame is attached to one (target) of the satellites in this maneuver [54]. The most used model for this mission in spacecraft rendezvous is given by Clohessy-Wiltshire equations, which have been widely adopted to study the spacecraft relative motion problems. By assuming small distance between the chaser and the target, the linearized equations of the relative motion between them can be described as below [13], [54]:

$$
\left(\begin{array}{c}
\ddot{x}-2 n \dot{y}-3 n^{2} x \\
\ddot{y}+2 n \dot{x} \\
\ddot{z}+n^{2} z
\end{array}\right)=\left(\begin{array}{c}
\gamma_{x} \\
\gamma_{y} \\
\gamma_{z}
\end{array}\right)
$$

where $n$ denotes the mean motion of the target vehicle. These equations can be used to study the forces required to perform an orbit rendezvous, the displacements from a reference trajectory produced by maneuvers or other velocity changes and the effects of perturbations on the displacements from a reference trajectory. These second-order differential equations are valid for small displacements (a few tens of kilometers in the radial and out-ofplane directions) but remain correct for an order of magnitude (hundreds of kilometer) of larger change in the down track coordinate. Several articles can be referred to as samples of rendezvous missions for additional information [55], [18].

The Clohessy-Wiltshire equation set is derived from the assumptions that the two spacecraft run on neighboring two-body circular orbits and the relative distance between the two spacecraft is much shorter than their geocentric distance. Moreover, first-order approximations are used so that second- and higher-order terms of relative positions and velocities are ignored. It needs improvements in order to describe relative trajectories not satisfying these assumptions. For the sake of brevity, the detailed description of improved relative dynamics equations for space rendezvous is omitted here and the reader is referred to the survey by Luo et al. in 2014 [4]. 


\subsubsection{Libration points}

Libration points, sometimes referred as Lagrange's points, are essentially the gravitational equilibrium in celestial mechanics, where a spacecraft is able to keep stationary with respect to the primary and secondary bodies without fuel consumption. Therefore, they can motivate numerous space missions due to their special locations. Simulation of transfers to these points needs models based on N-body problem since the gravitational force of more than one celestial body is considered on the spacecraft. The trajectories used by these missions are solutions of the Circular Restricted Three-Body Problem (CR3BP). CR3BP is the simplest model to study the three-body problem, as well as the most useful one to investigate the motions and phase space structure near libration points. However, it is sometimes not accurate enough for astronautical applications.

The planar CR3BP describes the motion of a spacecraft moving in the gravitational field of two primaries $P_{1}$ and $P_{2}$, with masses $m_{1}$ and $m_{2}$. The equations of motion in the normalized synodic reference frame, are [44]:

$$
\left(\begin{array}{l}
\ddot{x}-2 \dot{y} \\
\ddot{y}+2 \dot{x}
\end{array}\right)=\left(\begin{array}{l}
\Omega_{x} \\
\Omega_{y}
\end{array}\right)
$$

with the effective potential given by

$$
\Omega(x, y)=\frac{1}{2}\left(x^{2}+y^{2}\right)+\frac{1-\mu}{r_{1}}+\frac{\mu}{r_{2}}+\frac{\mu(1-\mu)}{2}
$$

and

$$
\begin{aligned}
\mu & =\frac{m_{2}}{m_{1}+m_{2}}\left(m_{2}>m_{1}\right) \\
r_{1} & =\sqrt{(x-\mu)^{2}+y^{2}} \\
r_{2} & =\sqrt{(x+1-\mu)^{2}+y^{2}}
\end{aligned}
$$

where $r_{1}$ and $r_{2}$ denote the distances from the particle to $P_{1}$ and $P_{2}$, respectively, and $\mu$ (not to be confused with the gravitational constant in previous subsections), known as the mass parameter of the CR3BP, is the dimensionless mass of $P_{2}$. The normalized variables are such that the distance between $P_{1}$ and $P_{2}$, the sum of their masses, and their angular velocity around the barycenter are normalized to one. So, one complete rotation of the primaries 
around their barycenter with respect to an inertial frame occurs in $2 \pi$ dimensionless units of time, and, in the synodic frame, $P_{1}$ and $P_{2}$ are fixed at $(\mu, 0)$ and $(\mu-1,0)$, respectively. Halo orbits located around the collinear libration points in the CR3BP can be well established regarding this modeling. See [56] and [57] for some instances.

The presented dynamic equations are suitable for the $2 \mathrm{D}$ problem. The $3 \mathrm{D}$ form of CR3BP has one additional differential equation for $\mathrm{Z}$ axis. Besides, the center of the main coordinate system may be modified and shifted toward different masses in some research. The general 3D representation of CR3BP is as follows:

$$
\left(\begin{array}{c}
\ddot{x}-2 \dot{y}-x \\
\ddot{y}+2 \dot{x}-y \\
\ddot{z}
\end{array}\right)=\left(\begin{array}{c}
-\frac{(1-\mu)(x+\mu)}{r_{1}^{3}}-\frac{\mu(x-1+\mu)}{r_{2}^{3}} \\
-\frac{(1-\mu) y}{r_{1}^{3}}-\frac{\mu y}{r_{2}^{3}} \\
-\frac{(1-\mu) z}{r_{1}^{3}}-\frac{\mu z}{r_{2}^{3}}
\end{array}\right)+\left(\begin{array}{l}
\gamma_{x} \\
\gamma_{y} \\
\gamma_{z}
\end{array}\right)
$$

Numerous researches use this model for libration points [58]. The more general representation of system dynamics for Lagrange's points is the extended continuous thrust form for N-body problem in the Earth-centered inertial coordinate frame as [13]:

$$
\vec{r}=-\frac{-\mu_{E}}{r^{3}}+\sum_{i=1}^{N} \mu_{i}\left(\frac{\overrightarrow{r_{i}}-\vec{r}}{\left|r_{i}-r\right|^{3}}-\frac{\overrightarrow{r_{i}}}{r_{i}^{3}}\right)+\vec{\Gamma}
$$

where $\mu_{i}$ is the gravitational constants for any of the planets considered in the model, while $\overrightarrow{r_{i}}$ is the position vector for that planet. An example of this model is used in [59], which considers the solar radiation pressure as the perturbed acceleration.

Other forms of equations describing the dynamics of Lagrange's points vary for different coordinate systems and expected accuracies. One representation is the Elliptical Four-body Problem (EFBP) in which the Moon moves around the Earth in an elliptical motion and the Earth-Moon system moves around the Sun in a circular orbit. All the central bodies are in the same plane. Although the EFBP is not the most faithful model for the spacecraft, it does include the most important facts, the Sun's direct influence and the Moon's elliptical motion, which cannot be analyzed in the Circular Restrict 
Three-Body Problem (CR3BP). More details about mathematical models for libration points can be found in [60].

\subsection{Summary}

In spacecraft trajectory optimization, modeling the engineered system dynamics is the primary step. The model of the dynamic system is a set of equations (differential equations) that represents the dynamics of the system using laws of physics. The model allows the study of spacecraft transients and steady state performance. Examples of models in spacecraft trajectory optimization are tabulated in Table 4.

\begin{tabular}{cc}
\hline \hline Mission & \multicolumn{1}{c}{ References } \\
\hline Inertial coordinates & {$[61][62][63][64][65][66][67]$} \\
\cline { 2 - 2 } Modified equinotical elements & {$[68][69][70][71][50][51][72][73]$} \\
Rendezvous & {$[74][75][76][77][78][79][80]$} \\
\cline { 2 - 2 } Libration & {$[81][82][83][84][20][18][85][86]$} \\
& {$[87][72][88][85][89][90][91][92]$} \\
\hline
\end{tabular}

Table 4: Continuous model in spacecraft trajectory optimization

As the model becomes more detailed, it also can become more accurate. Model accuracy needed for spacecraft trajectory optimization is normally simpler than the model used for system simulation. Ignoring some physical phenomena, linearly approximating nonlinear characteristics and using the approximation of lumped parameters in spacecraft are the ways which turn complex models to simple ones for trajectory optimization.

\section{Objective}

The second key element of the spacecraft trajectory optimization process is defining objectives based on the space mission requirements. Objectives are defined by means of some functions, usually referred to cost functions in optimal control terminology or objective functions in computer science terminology. They may comprise fuel mass, total velocity increment, state errors, transfer time, or acceleration. Besides, other components may be also considered in some space missions due to the employment of specific techniques or mission criteria. 
Although the objective functions vary from problem to problem, its general form, better known as Bolza cost function [11], can be defined as follows [12]:

$$
J(\vec{x}, \vec{u}, t)=h\left(\vec{x}\left(t_{f}\right), t_{f}\right)+\int_{t_{0}}^{t_{f}} g(\vec{x}(t), \vec{u}(t), t) d t
$$

where $t_{0}$ and $t_{f}$ are the initial and final times, respectively. The function $h$ represents the Mayer term which denotes the cost related to the final states and $g$ is referred to as the Lagrange term or the running cost which tracks the state and control costs that occur through their entire time histories. This general form of the objective function represents a complete cost function in optimal control theory. The objective function may contain just the Mayer term, just the Lagrange term, or both, depending on what is being optimized in space travel. Various forms of objective function can be categorized in two different aspects, including type and quantity as illustrated in Fig. 4.

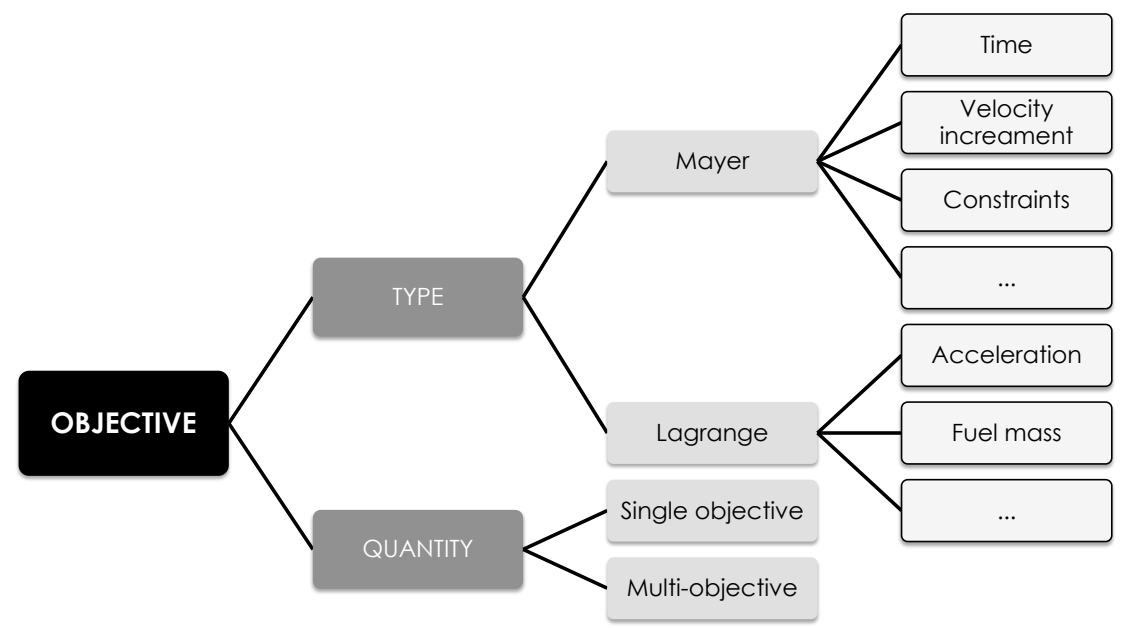

Figure 4: Taxonomy of objectives in spacecraft trajectory optimization

One common and familiar definition between all types of objective functions is presented by Conway [2]. It states that the objective of any space mission depends on two concepts, including having minimum time or minimizing control effort, referring relatively to the Mayer and Lagrange terms in Eq. 31. This type of definition can be considered as a subcategory for the type of the objectives. As for the number of objectives, cost functions can be roughly divided into single objective and multi-objective ones [93]. As will be discussed in this section, the simplest way to deal with multipurpose 
spacecraft optimization problem is to consider an overall objective function being the weighted sum of the single objective functions [94].

The following subsections are dedicated to the different types of objective functions according to the taxonomy, ending with a brief discussion about the typical techniques for dealing with multi-objective problems.

\subsection{Mayer}

The first types of objectives are those which are related to the Mayer term. These objectives are functions of state variables at the end of a transfer trajectory.

\subsubsection{Time}

The time in spacecraft trajectory optimization problems is simply the transfer time in space travels. While the control effort has different kinds of representations as previously mentioned, such as fuel mass, thrust level or acceleration, the time has a simple representation in most of the problems. For minimum time problems, the cost function can be simply defined as:

$$
J=t_{f}
$$

where $t_{f}$ is the transfer time. One example regarding the use of this cost function is the trajectory optimization of libration points in Earth-Moon system, in which the duration of flight to perform the mission purposes is expected to be minimized [95]. Shang et al. [96] used this cost function to minimize the transfer time for a Earth to Mercury space mission utilizing solar sails.

\subsubsection{Velocity increment}

In many spacecraft trajectory optimization problems the objective is to minimize the velocity increment or the summation of the increments in multiple phases. A typical example is the well-known multiple gravity assist mission with $n$ stages [34]. In this problem each impulse causes a mass consumption proportional to the modulus of the change of velocity. Therefore, in order to minimize the overall mass consumption, the following objective function is usually considered:

$$
J=\sum_{i=0}^{n}\left|\Delta v_{i}\right|
$$


where $\Delta v_{i}$ is the single change of velocity dedicated to each trajectory between two astronomical body (planets or asteroids). This representation of cost function is very popular specifically for multi-impulse problems [22], [16] and even rendezvous missions [55], [18].

\subsubsection{Initial and terminal conditions}

Although the terminal conditions (or even in some cases the initial conditions) are generally considered as constraints, there are some researches which deal with these constraints as objectives [97]. The general form will be as follows:

$$
J=\phi\left(\vec{x}\left(t_{0}\right), \vec{x}\left(t_{f}\right)\right)
$$

where $\phi$ is the initial and terminal constraint. Note that this function is more general than the function $h$ given at Eq. 31. This kind of cost function is used in several articles including gravity assist maneuvers [50], [51], continuous interplanetary trajectories [96], spacecraft reentry [98] and orbit rising mission [99].

\subsection{Lagrange}

Unlike the objectives related to the Mayer term, the second types of objectives are functions in the form of Lagrange term in Eq. 31. These costs are integrals of inputs or state variables in the transfer trajectory.

\subsubsection{Acceleration}

One cost function which is usually considered to be minimized in space missions is the integration of the square of spacecraft acceleration within the transfer trajectory. Its representation is as follows:

$$
J=\frac{1}{2} \int_{t_{0}}^{t_{f}} \gamma^{2} d t
$$

where $\gamma$ is the magnitude of the spacecraft acceleration, typically due to the propulsion system, while $t_{0}$ and $t_{f}$ are the initial and final time of spaceflight. This representation is popular in researches including thrust minimization [100], [71]. Other forms of cost functions for acceleration are popular in spacecraft trajectory optimization problems, specifically in shape-based techniques [101]. The reason is that in these researches, the state vectors are directly interpolated via polynomials with discretization [59]. So the acceleration (similarly the thrust magnitude) will be achieved as a function of 
optimization variables. In such cases, the total velocity increment can be calculated by integrating the norm of the acceleration vector as:

$$
J=\Delta v=\int_{t_{0}}^{t_{f}} \sqrt{\gamma_{x}^{2}+\gamma_{y}^{2}+\gamma_{z}^{2}} d t
$$

where $\gamma_{x}, \gamma_{y}, \gamma_{z}$ are the components of overall acceleration of the spacecraft within the transfer trajectory. Obviously, these components can be substituted by any coordinate system [102]. In some researches [103], acceleration is sometimes represented by $T / m$ as follows:

$$
J=\int_{t_{0}}^{t_{f}} \frac{T}{m} d t
$$

where $T$ and $m$ are the thrust magnitude and the mass of the spacecraft, respectively. In this case, the maximum value or the integral of thrust magnitude will be considered as the cost function. Eq. 36 along with Eq. 33 are both representations of velocity increment in discrete and continuous domains, respectively. The type of the model and the approach determine which one should be used in spacecraft trajectory optimization.

\subsubsection{Fuel mass}

Besides velocity increment and acceleration, sometimes fuel mass is considered as an alternative representation of energy. The study of fuel cost function for spacecraft trajectories has been taken into consideration for a long time [104]. One representation is as below.

$$
J=\int_{t_{0}}^{t_{f}} m_{f} d t
$$

where $m_{f}$ is the fuel mass of the spacecraft within the transfer trajectory. If the transfer time and the mass decreasing rate is fixed, the fuel mass will be independent of the transfer trajectory and can be calculated directly. In such cases, the fuel mass takes the Mayer form simply as $J=m_{f}$, as in [45]. Also, in some research the fuel mass itself is an input of the optimization process which turns this variable into a known and fixed parameter in the overall process. 


\subsection{Other objectives}

Besides the common objectives, sometimes additional costs are considered in some problems, depending on the approach or mission criteria. For example, Luo and Tang [105] employed the following cost function in order to prevent the solution from having a non-smooth trajectory:

$$
J=\frac{1}{2} \int_{t_{0}}^{t_{f}}[\vec{x}(t)]^{T} Q[\vec{x}(t)] d t
$$

where $Q$ is the state weighting coefficient. Employment of this cost function, besides other familiar cost functions, overcomes the production of a nonsmooth or badly scaled trajectories.

Optimization of multi-spacecraft constellations problem by Li et al. [106] is another example of employing a special cost function. Since the problem in this research is formation flying, a very specific cost function including several terms is considered. Objectives such as collision, path length, execution time, fuel consumption and fuel distribution are considered simultaneously.

As another example in the multi gravity assist trajectory optimization problem, the constraints on the minimum distance from the center of each planet can be included in the objective function through appropriate penalty terms [24]. Therefore, some modifications are applied to the objective function. Vasile and Locatelli [34] considered the following modification to the typical cost function in that problem:

$$
J=J_{0}+\sum_{i=1}^{n} w_{i} \max \left[r_{i}-\gamma_{i}\right]
$$

where $J_{0}$ is a typical cost function in the form of Eq. 33, $w_{i}$ are given penalty parameter values and $\gamma_{i}$ are the pericentre radius of planets in $n$ stages of the space mission. This form of objective function has also been used in [107].

\subsection{Scalarization}

As stated, objectives can also be categorized according to their number. Single-purpose methods attempt to determine the solution taking into account a single criterion, whereas multi-purpose methods search for a trade-off among several distinct criteria. Almost every advanced spacecraft trajectory optimization problem involves multiple conflicting criteria and it is not possible to mathematically define a single optimal solution. However, a set of 
compromises called Pareto optimal solutions can be defined. In such problems, there is no unique solution that optimizes both objectives [2]. For instance, in order to force the optimization to meet the boundary conditions while searching the proper set of inputs for the maximization or minimization of the given cost function, it is convenient to attempt to minimize an augmented cost function. This process, better known as scalarization [108], is the method which transforms the problem into a single-objective optimization one involving possibly some parameters or additional constraints. Separate terms in the cost function are given appropriate weights designating their relative importance in the optimization. This is perhaps the most difficult part of designing the cost function. There are an infinite number of weighting combinations if multiple terms are present. One representation of using a weighting coefficient in a general form is presented in [109] as the following:

$$
J(\vec{x}, \vec{u}, t)=h\left(\vec{x}\left(t_{f}\right), t_{f}\right)+\alpha \int_{t_{0}}^{t_{f}} g(\vec{x}(t), \vec{u}(t), t) d t
$$

where $\alpha$ denotes the relative importance of minimizing cost to terminal conditions. The primary purpose of this weighting factor is to balance the cost function such that the Mayer and Lagrange terms have the same relative order of magnitude. It has been stated that for the impulsive cases where the thrust time is small relative to the scenario time, it required a weighting factor on the order of $10^{-2}$. For the continuous case the thrust time was larger relative to the total scenario time requiring this weighting factor to decrease to the order of $10^{-6}$ [109]. However, each scenario requires specific manipulation of this variable in order to properly balance the cost function.

The rest of the methods in scalarization techniques are more practical than Eq. 41. In these techniques, the Mayer and Lagrange terms in the main equation are separated into several minor cost functions $J_{1}, J_{2}, \ldots J_{p}$ for $p$ number of minor cost functions. It allows the user to specify preferences, which may be articulated in terms of goals or the relative importance of different objectives [94]. Most of these methods incorporate parameters, which are coefficients, exponents, constraint limits, etc, that can either be set to reflect decision-maker preferences, or be continuously altered in an effort to represent the complete Pareto optimal set. Some forms of modified cost functions in spacecraft trajectory optimization problems are as follows:

$$
J=\sum_{i=1}^{n} J_{i}
$$




$$
\begin{gathered}
J=\sum_{i=1}^{n} \alpha_{i} J_{i} \\
J=\sum_{i=1}^{n} \frac{J_{i}}{\alpha_{i}} \\
J=\sum_{i=1}^{n} J_{i}^{2}
\end{gathered}
$$

where in some of them each cost function is multiplied or divided by a weighting coefficient. Some literature in which the spacecraft trajectory optimization problems are solved employing these representations is provided in Table 5.

\begin{tabular}{ccc}
\hline \hline Scalarization & Modified cost function & References \\
\hline Sum method & $J=\sum_{i=1}^{n} J_{i}$ & {$[105][107][69][72][110]$} \\
Weighted sum method & $J=\sum_{i=1}^{n} \alpha_{i} J_{i}$ & {$[109][58][111][112][86]$} \\
Normalized method & $J=\sum_{i=1}^{n} \frac{J_{i}}{\alpha_{i}}$ & {$[113][45]$} \\
Global criterion method & $J=\sum_{i=1}^{n} J_{i}^{2}$ & {$[114][61]$} \\
\hline \hline
\end{tabular}

Table 5: Scalarization techniques in spacecraft trajectory optimization

Choosing proper weighting coefficients in this form is a challenging issue and has been tackled in different ways in various researches. For instance, Bolle and Circi [113] tackled this problem by choosing the maximum propagation time allowed in the simulation as the related coefficient for the time cost function and manually tuning the other weighting coefficient (magnitude of the error tolerance permitted during the mission design phase) in order to make both cost functions behave in the same order. As such, extreme care must be taken in properly balancing the relative weights in the cost function. For fundamental background in the associated multi-objective optimization, the reader should refer to Marler and Arora [93]. 


\section{Approach}

Hitherto, the spacecraft dynamics and the objectives have been modeled and properly defined for the spacecraft trajectory optimization problem. The problem can now be introduced as a general representation as depicted in Fig. 5. Details for the dimensions of variables are provided in [115]. The next step in spacecraft trajectory optimization is to develop an approach for finding the optimal trajectory. Since this step is a vast subject, only an overview of approaches with a brief discussion is provided in this section. For a fundamental background in the associated approaches, the reader should refer to $[10]$.

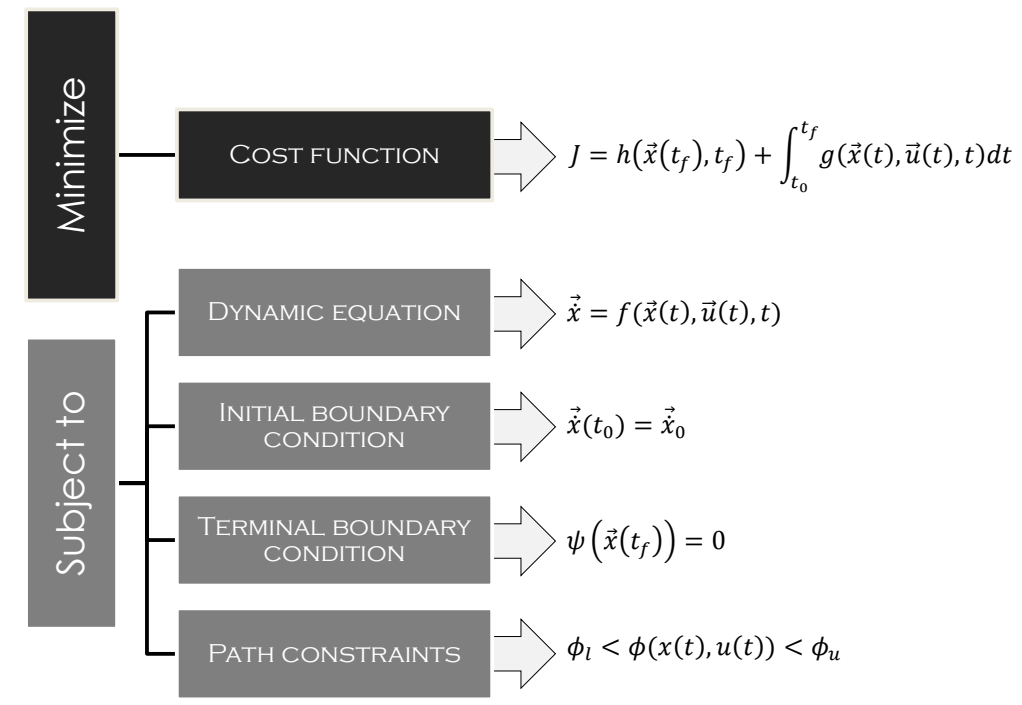

Figure 5: Mathematical representation of a spacecraft trajectory optimization problem

While proceeding to the details in this section, the distinction between the terms approaches, methods and techniques will be provided in order to avoid misunderstanding. Each of these terms include a specific bunch of ideas which are illustrated in the map of trajectory optimization approaches as in Fig. 6 .

In general, two types of approaches exist: analytical approaches and numerical approaches. Analytical approaches for the optimal trajectory result in analytical solutions. They can only be obtained in special cases, for example for very low-thrust orbit raising [116], and sometimes in the presence of some perturbations [55]. Therefore, results from the analytical approach are 


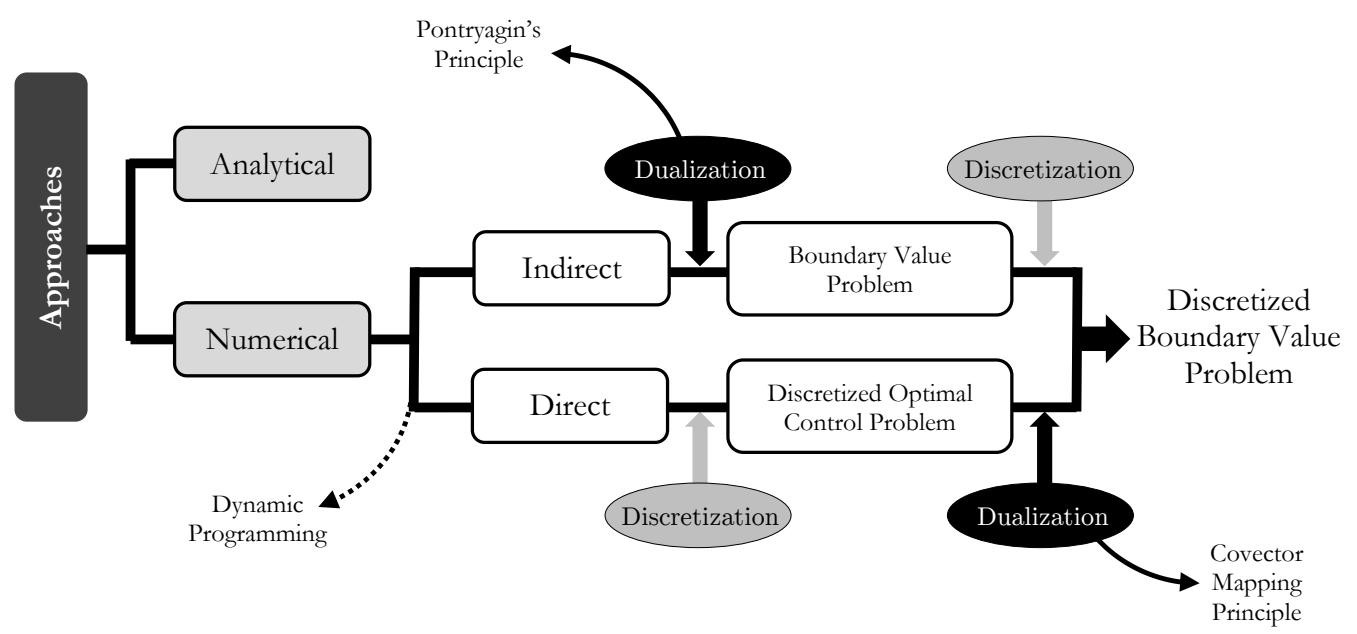

Figure 6: Taxonomy of approaches in spacecraft trajectory optimization

seldom feasible for most of the spacecraft trajectory optimization problems [2].

The majority of researches are dedicated to numerical approaches for spacecraft trajectory optimization problems [2]. These approaches can be divided in two well-known methods, called direct and indirect methods [10], [117]. Through direct methods, the solution is found in an approximate way based on the concept of parameterization on state variables $\vec{x}(t)$ and control inputs $\vec{u}(t)$. The parameterization concept usually involves discretization which concerns the process of transcription of the problem for transferring continuous functions, models, and equations into discrete counterparts. Certainly, such methods allow a candidate solution to be found, but no warranty is given about its optimality [10]. Indirect methods, on the other hand, use the same techniques and concepts as in the direct method, but have the feature of relying on necessary, analytic conditions for optimality. This allows the shifting of the optimization problem onto the determination of some parameters, known as the Lagrange multipliers, that should fulfill conditions for optimality at the beginning and at the end of the process. These variables are often defined as co-state, as they evolve along with the state vector. In other words, the main difference between direct and indirect methods is the involvement of co-state variables. The main issue related to indirect methods is the impossibility of knowing the initial guess, permitting the meeting of the boundary conditions. The search domain in such methods which is based 
on Pontryagin's Principle [2], is made even more complex by the fact that each Lagrange multiplier ranges in an unbounded set [10]. The connection between these two methods has been a challenge and a matter of interest within the community. The long lasting history regarding the bond of these methods has resulted in the recent advance in trajectory optimization [118]. This connection is described with respect to the Covector Mapping Principle, thanks to the efforts of Ross and Fahroo [119], [120], [121]. It describes the relationship between the multipliers of the discretized optimization problems and the co-states of the continuous optimal control problem.

Besides these two categories, sometimes dynamic programming is also considered as the third branch of numerical methods (dashed line in Fig. 6) in which the optimality criteria in continuous time is based on the HamiltonJacobi-Belman partial differential equation. However, most research in literature only consider direct and indirect methods as the only two branches of the numerical approaches [1], [2]. An overview of the approaches mentioned along with their related methods, techniques and theories will be briefly discussed in this section.

\subsection{Analytical approaches}

Analytical approaches are the most desired ones since they usually provide solutions based on mathematical representations directly with zero approximation. However, they are not achievable most of the time due to the complexity of the problem. Such kinds of complexities may be because of the mathematical model or the objectives. The primary example of an analytical approach is the well-known Hohmann transfer for a simple orbit transfer mission. According to the proposed terminology and taxonomy in this review, Hohmann transfer is actually a very simple approach for transferring the spacecraft from one orbit to another for velocity increment minimization.

As for the analytical approach in a continuous domain, generally the process of achieving the optimal solution involves optimal control theory and relies on Pontryagin's Principle. The first step is establishing the problem in mathematical representation. This consists of determining the equations of motion, cost function, and applicable constraints. Constraints can be broken down into two primary types: path constraints and boundary constraints [122]. Path constraints represent restrictions on either the control or state at any time. For instance, engines have a finite amount of thrust yielding a maximum value for the control. It would be meaningless to look for a solution that requires a thrust more than the maximum available limit. 
Boundary constraints pertain to either the final or initial states. They may be given as a set of equality or inequality constraints. A state vector that does not violate any constraint is referred to as an admissible trajectory. Similarly, a control vector that does not violate any constraint is referred to as an admissible control. Then, the cost function is formed, augmented with Lagrange multipliers (or co-states) associated with the constraints and state differential equations of the system. Defining a convenient Hamiltonian, the first variation of the cost function due to differential changes in the control inputs is written. Next, co-state differential equations and boundary conditions are chosen to simplify this expression. This process of writing a problem in terms of the original variables and Lagrange multipliers (or states and co-states) is often referred to as dualization which makes the problem difficult to solve analytically. Such difficulties are well described by Ross [11]. However, this analytical approach is very useful as a sanity check for the numerical approaches.

As is shown in many references [10], [11], the number of Lagrange multipliers equals that of the state vector components. This means that even in the simplest case by considering the spacecraft as a material point, thus ignoring the attitude equations, the optimal set of Lagrange parameters could potentially be searched in $\mathcal{R}^{7}$ (six entries for the spatial coordinates plus one for the spacecraft mass). If even the initial epoch of the transfer is to be determined, the optimal set of parameters must be searched in $\mathcal{R}^{8}$.

Notable researches are dedicated to analytical approaches in spacecraft trajectory optimization. For example, Fernandez [123] developed a complete first-order analytical approach for the problem of optimal low-thrust limitedpower transfers in an inverse-square force field between coplanar orbits with small eccentricities. The presented approach eliminates the singularity for circular orbits and can be applied for time-fixed transfers between coplanar orbits with small eccentricities.

\subsection{Numerical approaches}

By increasing the complexity of the model and the problem, the analytical approaches fade and numerical approaches become more favorable. A few of the popular methods in numerical approaches are discussed here, following the surveys [1] and [2].

\subsubsection{Direct and indirect methods}

The overall schema of numerical approaches is depicted in Fig. 7. 


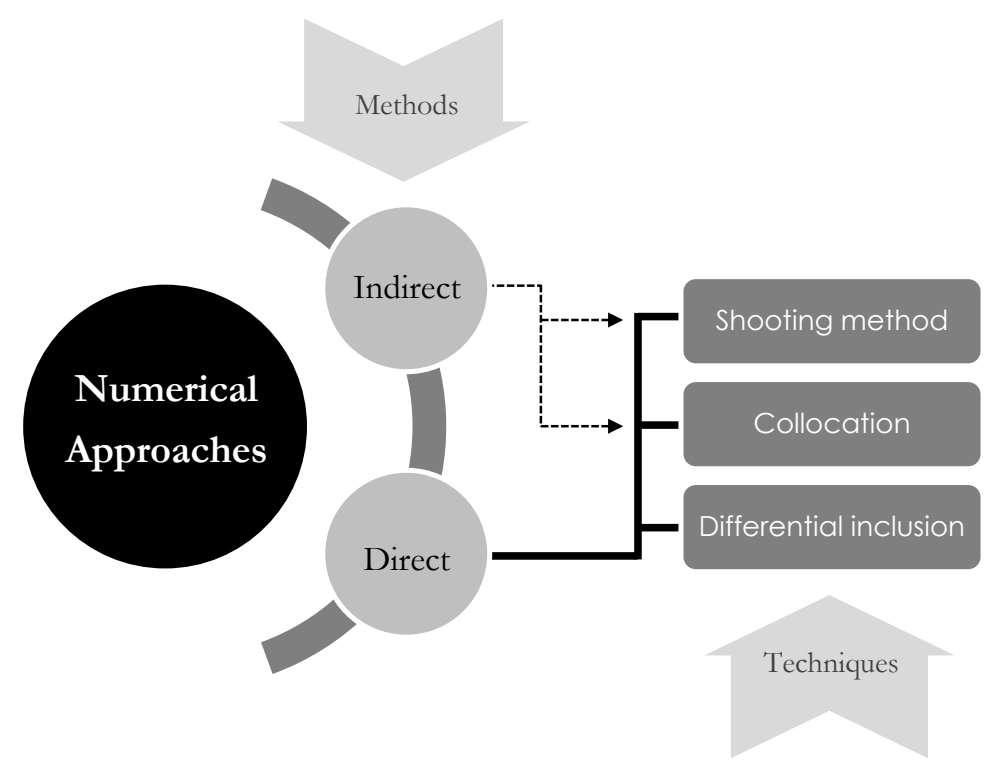

Figure 7: Methods and techniques in numerical approaches

Two distinct branches of numerical approaches have arisen. Both branches attempt to minimize cost functions and constraint violations using discrete approximations [124]. This is performed by some gradient-based or metaheuristics which will be discussed in the next section. The first branch is that of direct methods which transcribe the continuous optimal control problem into an optimization problem [9] considering states and control vectors. Satisfaction of the system equations is accomplished by integrating them stepwise using either implicit or explicit rules; in either case, the effect is to generate nonlinear constraint equations which must be satisfied by the parameters, which are the discrete representations of the state and control time histories. The problem is thus converted into a nonlinear programming problem [125]. Although direct methods are less accurate than indirect methods, the fact that they are easier to implement, have a larger domain of convergence, and have reduced problem size make them very attractive. One type of direct methods is the one when only the state variables are interpolated and control variables are considered in the objective function. Then a gradient-based technique or metaheuristic is employed to minimize the cost by changing the values of state variables. This method is sometimes referred to as the shape-based method, since it pertains to the shape 
of the state variables. Fourier series are very popular in this kind of method, specifically when applied to low-thrust trajectory optimization [47], [101], [71],[126]. The trajectory determined by the shape-based methods satisfies the equation of motion, boundary conditions, and even the constraint on the thrust acceleration. In addition, the solution of the shape-based methods can be shown to serve as a good initial guess for other approaches or methods. The resulting approach is very popular since it can be used in spacecraft trajectory optimization problems considering constraints or any other type of limitations. They have advantage as they can be easily manipulated for overcoming drawbacks of other methods. For instance, Xie et. al [48] used the shape-based approach in providing a new combination of the elevationangle and radius shapes for the 3-D low-thrust trajectories using the initial orbital plane as the reference plane. This new shape combination avoids the two drawbacks of the spherical shaping method, including large out-of-plane motion and range control of state angle variation.

The second branch is that of indirect methods. An indirect method considers the dualized form of the equations including states and co-states within the time discretization. That is, the states and co-states are both considered within the discretization. While indirect methods typically enjoy greater accuracy than direct methods, three major problems arise. Firstly, the analytical forms of the necessary conditions must be expressed, including the co-state differential equations, the Hamiltonian, the optimality condition, and transversality conditions. Numerically speaking, this also makes the problem size large due to discretization of the co-states. Secondly, the analyst must guess certain aspects of the solution, such as the portions of the time domain containing constrained or unconstrained control arcs. Finally, this method also requires initial guesses for the co-states which decreases the domain of the convergence [9].

Previously mentioned, the very rarely considered branch is the dynamic programming method, which is seldom considered as a separate subdivision of numerical approaches. The basic idea is to subdivide the problem to be solved in a number of stages. Each stage is associated with one subproblem, and the subproblems are linked together by a recurrence relation. The solution of the whole problem is thus obtained by solving the subproblems using recursive computations. For a more detailed insight in dynamic programming, the reader is referred to [127]. Dynamic programming has been extensively applied with success to discrete problems. Unfortunately, its application is severely restricted in the case of continuous state systems because 
of the curse of dimensionality; a term coined by Bellman to describe the problem caused by the exponential increase in the size of the state space [8]. Therefore, dynamic programming has not been successfully used in spacecraft trajectory problem with a large number of variables. Other methods, such as direct or indirect methods, must be employed. It should also be noted that regardless of whether a direct or indirect method is chosen, the states must be integrated from some boundary condition or the equations of motion must be enforced through constraints.

Both of the aforementioned direct and indirect methods aim at a highfidelity solution, but may be time consuming for evaluating thousands of trajectories in the preliminary phase of the mission design. There have been various efforts and routes taken in overcoming the difficulties associated with the design of optimal spacecraft trajectories, for instance, by resorting to heuristics.

\subsubsection{Numerical techniques}

Hitherto, two different methods have been introduced within the category of numerical approaches. In this subsection, some numerical techniques will be briefly introduced which can be used in direct and indirect methods.

\section{Differential inclusions}

One strictly direct technique is that of differential inclusions. The differential inclusions enforce the equations of motion at each discrete time by applying inequality constraints on the state derivatives [128]. These inequality constraints are obtained by substituting the upper and lower bounds on the control vector into the equations of motion. When the inequality constraints are met, the states at one node are said to lie in the attainable set at that node, given the state values at an adjacent node and the set of admissible controls. The advantage given by differential inclusions is that it effectively eliminates the explicit dependence on control values at each node. However, techniques such as this can become numerically unstable and the formulation can be problem dependent [1].

\section{Shooting methods}

The shooting method is a well-known iterative technique to calculate the state histories given the control histories of the system. Most successful shooting applications have one salient feature in common, namely, the ability to describe the problem in terms of a relatively small number of optimization variables. One example is [96], where the direct shooting method is employed with an evolutionary algorithm to solve the minimum-time orbit transfers of 
solar sail spacecraft for Mercury sample return missions.

Shooting methods can be divided into two types including shooting method and multiple-shooting method. The direct shooting method is a control parameterization method where the control is parameterized using a specified functional form, e.g.,

$$
u(t) \approx \sum_{i=1}^{m} a_{i} \psi_{i}(t)
$$

where $\psi_{i}(t)(i=1, \ldots, m)$ are known functions and $a_{i}(i=1, \ldots, m)$ are the parameters to be determined from the optimization. The dynamics are then satisfied by integrating the differential equations using a time-marching algorithm. Next, the cost function is determined using a quadrature approximation that is consistent with the numerical integrator used to solve the differential equations. The nonlinear programming problem that arises from direct shooting minimizes the cost subject to any path and interior-point constraints.

An extension of shooting the method is the multiple-shooting method. In a multiple-shooting method, the time interval $\left[t_{0}, t_{f}\right]$ is divided into $M+1$ subintervals. The aforementioned direct shooting method is then used over each subinterval $\left[t_{i}, t_{i+1}\right]$ with the values of the state at the beginning of each subinterval and the unknown coefficients in the control parameterization being unknowns in the optimization. In order to enforce continuity, the following conditions are enforced at the interface of each subinterval:

$$
x\left(t_{i}^{-}\right)=x\left(t_{i}^{+}\right)
$$

These continuity conditions result in vector root-finding problem, where it is desired to drive the values of the difference between $x\left(t_{i}^{-}\right)-x\left(t_{i}^{+}\right)$to zero. It can be seen that the direct multiple-shooting method increases the size of the optimization problem because the values of the state at the beginning of each subinterval are variables to optimize. This technique can also be applied in indirect approaches as well, where the co-states are also taken into account during discretization. The idea of the multiple-shooting method for both direct and indirect approaches is shown in Fig. 8 .

Despite the increased size of the problem due to these extra variables, the direct multiple-shooting method is an improvement over the standard direct shooting method. The sensitivity to errors in the unknown initial conditions is reduced since the integration is performed over significantly smaller time 


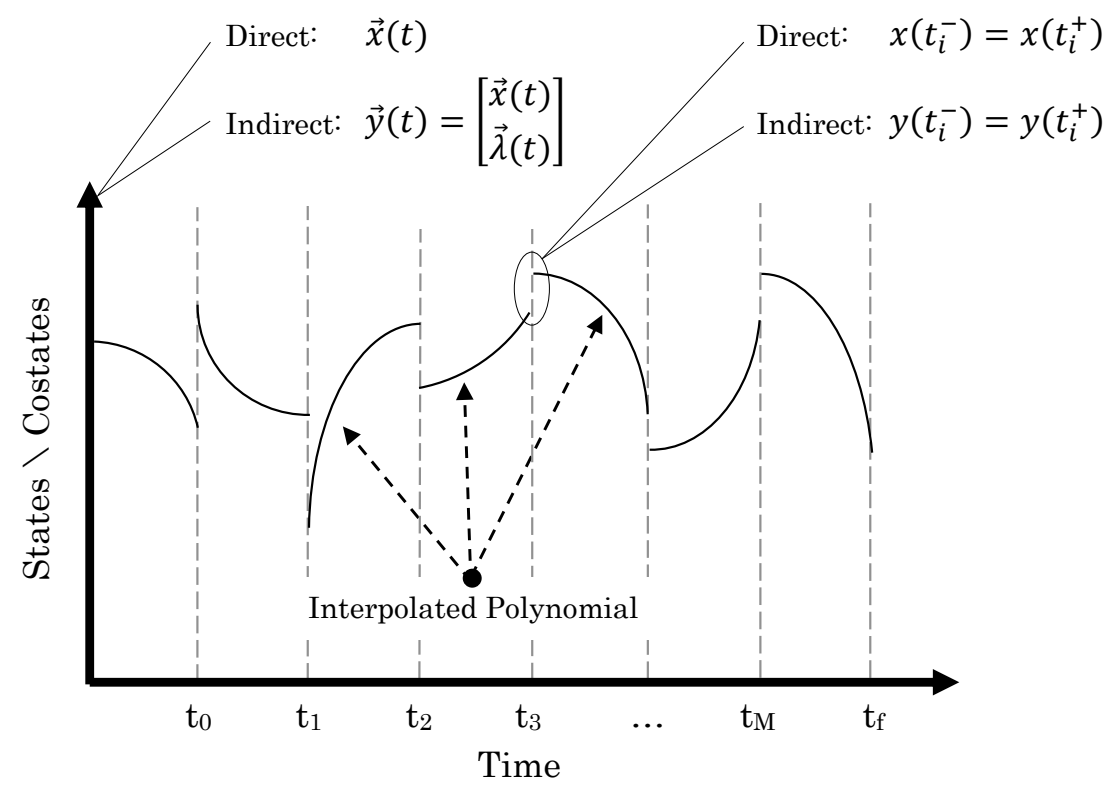

Figure 8: Diagram of direct and indirect multiple-shooting methods

intervals. Shooting methods are attractive because the equations of motion are enforced automatically by the marching integration. This effectively reduces the size of the problem by reducing the number of constraints that must be applied compared with collocation techniques which will be discussed in what follows [129].

\section{Collocation}

Collocation techniques enforce the equations of motion through quadrature rules or interpolation. See [130] as a typical example. An interpolating function (interpolant) is solved such that it passes through the state values and maintains the state derivatives at the nodes spanning one interval (or subinterval) of time. The interpolant is then evaluated at points between the nodes, called collocation points. At each collocation point, an equality constraint is formed, equating the interpolant derivative to the state derivative function, thus ensuring that the equations of motion hold (approximately) true across the entire interval of time. This technique, sometimes referred to transcription method [9] is based on a trajectory discretization by small segments and on a near-uniform discrete approximation of thrust directions by a set of thrust profiles with an inequality constraint for each segment. The problem in this case can be stated as to minimize the total character- 
istic velocity with terminal conditions. The overall scheme of this process is depicted in Fig. 9.

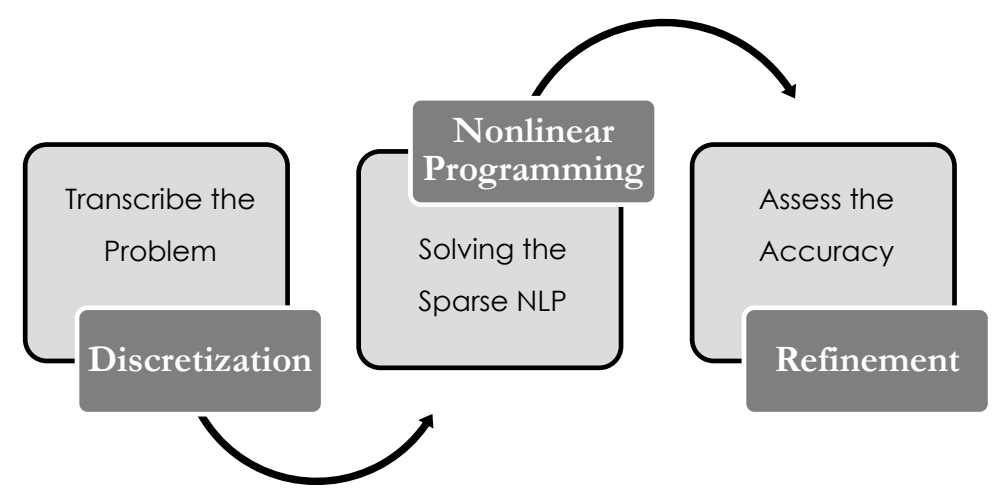

Figure 9: Transcription technique process

In fact, the technique can be considered as a sequential nonlinear programming algorithm. This process has three fundamental steps. The first step is to convert the dynamic system into a problem with a finite set of variables. The second step is solving the finite-dimensional problem using a parameter optimization method (i.e., the nonlinear programming subproblem). Finally, the third step is to assess the accuracy of the finite-dimensional approximation and, if necessary, repeat the transcription and optimization steps. Actually this technique is a process including an approach (discretization), a solution (NLP) and a minor technique (refinement). Details about these steps are provided in [9].

In this technique, one fundamental step is to discretize the spacecraft trajectory as depicted in Fig. 10. According to Fig. 10, trajectory discretization refers to a process which can be clearly seen as a multi-phase optimization problem. In this process, the trajectory is broken up into a finite number of legs and segments. The stage cost and constraints are generally expressed in terms of thrust magnitude and any violation from the maximum value. Transition functions can be obtained from the integration of the spaceflight equations of motion. The schematic representation of the corresponding trajectory structure is depicted in Fig. 11.

Once the states have been discretized and fitted with a polynomial, they are differentiated and then compared to the defined state derivatives at the collocation points. The difference is referred to as the defect. The defect is minimized in order to satisfy the specified equations of motion. This concept 


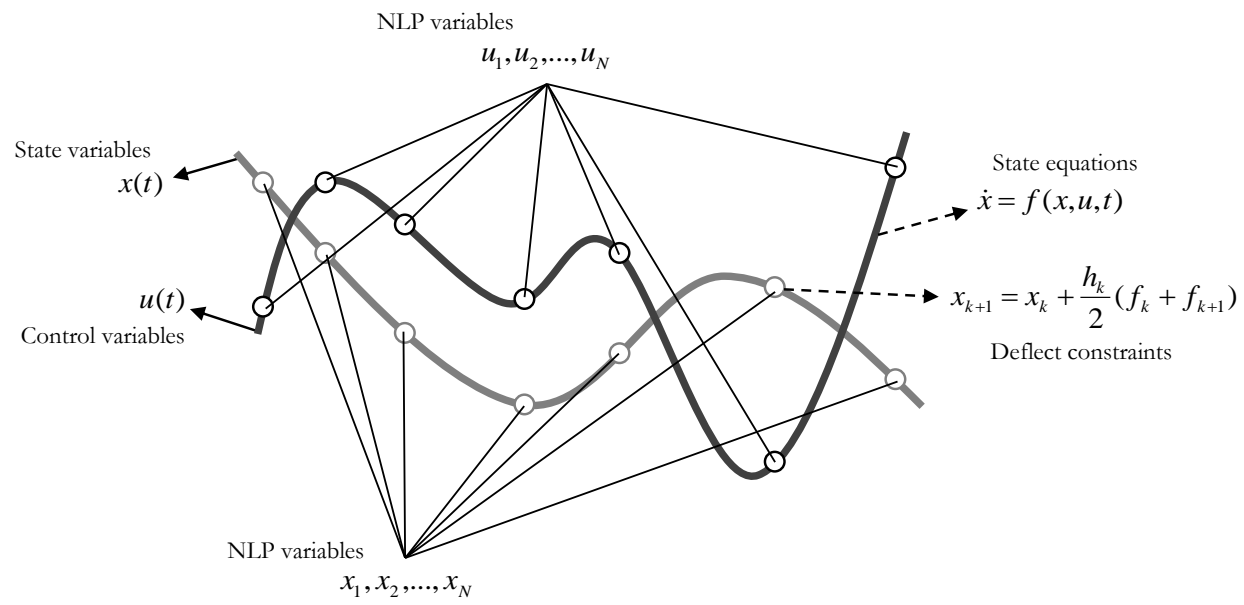

Figure 10: Discrete versus continuous trajectory concepts

is the main notion in a class of techniques called Pseudospectral methods. Pseudospectral methods represent a class of direct methods that use collocation to solve optimal control problems numerically rather than analytically. This technique has become increasingly popular over the past several decades. In a pseudo-spectral method, the collocation points are chosen based on accurate quadrature rules and the basic functions are typically Chebyshev or Lagrange polynomials. They are generally known to converge spectrally as mentioned in [131]. In these methods, the discretization is accomplished by the use of global polynomials at collocation points. There are many sets that are commonly used in pseudospectral methods such as Legendre-Gauss (LG), Legendre-Gauss-Radau (LGR), Legendre-Gauss-Lobatto (LGL), ChebyshevGauss-Lobatto (CGL) and Hermite-Legendre-Gauss-Lobatto (HLGL). Although some researchers prefer the term orthogonal collocation, the terms pseudospectral and orthogonal collocation have the same meaning [132]. The reader is referred to $[133,134,135,136,137,138,139,140]$ and references therein for recent and comprehensive reviews of pseudospectal methods along with their applications in trajectory optimization problems.

Needless to say, each method may be more appropriate under certain conditions. The accuracy of such discretizations has been compared in [99]. However, the accuracies of individual methods are not rigorously examined in this survey since the main focus is not on the collocation method itself. While space precludes a more detailed discussion of collocation methods, the 


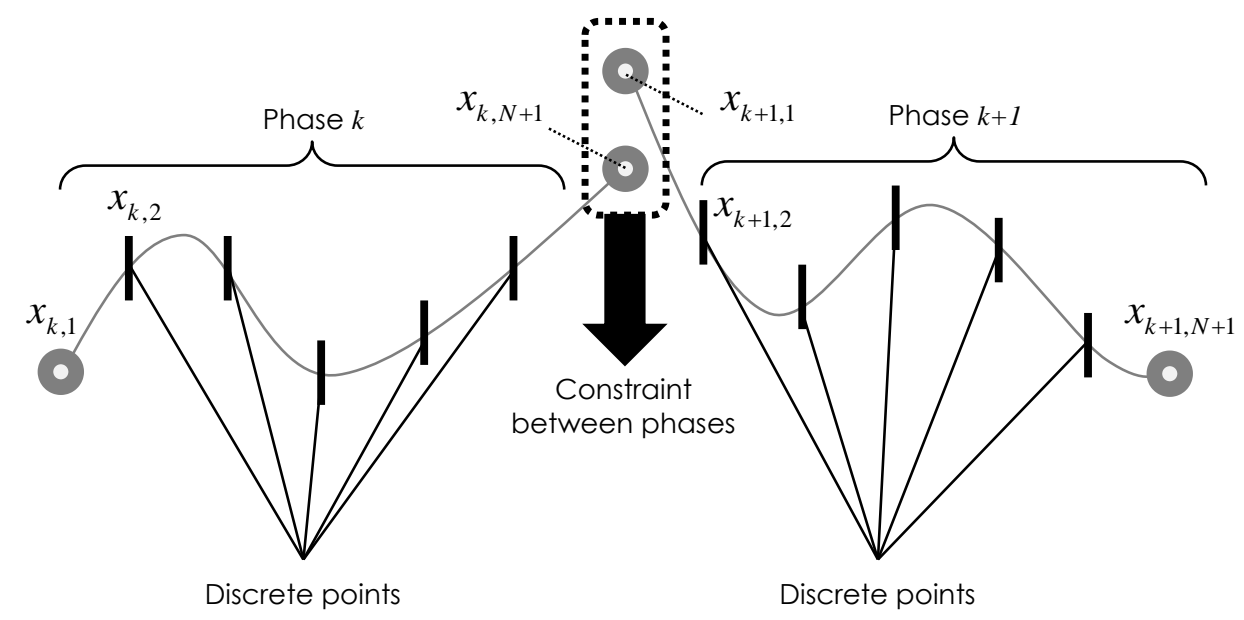

Figure 11: Spacecraft trajectory discretization

interested reader should consult the survey by Topputo and Zhang [141].

\subsection{Summary}

The approaches for spacecraft trajectory optimization problems are numerous and the current section is just a brief overview of current trends. As proposed in this section, when describing an approach for solving spacecraft trajectory optimization problems, it can be either analytical or numerical. It is possible to employ analytical approaches for specific space missions by a simpler process than the procedure identified in optimal control theory. One process is semi-analytic, which is often employed in academic-strength problems. They usually use clever coordinate transformations and other tricks to avoid relying on Pontryagin's Principle or mitigate the complexity of optimal control theory. While such ad hoc techniques are indeed useful and important for the analysis of specific problems, they are not portable to the broader spacecraft trajectory optimization problems. When turning into numerical approaches, a method is often classified as either a direct method or an indirect method. As stated, the direct methods transform the spacecraft trajectory optimization problem to large-scale problems that require a high number of iterative computations. It constructs a sequence of points such that the objective function is minimized. In an indirect method, the process is also the same. However, it attempts to find a root of the necessary condition based on Pontryagin's Principle. It means it focuses on the 
adjoint variables in addition to state and control variables. Contrary to popular belief, Pontryagin's Principle itself is not an approach for achieving the solution. It is a problem generator which maps the optimal control problem to a boundary value problem by lifting it to a dual space [11].

It is also important to emphasize that there is no restriction with the method used to solve the problem and the techniques. For example, with the exception of the differential inclusion, one may consider applying a shooting or multiple-shooting technique to either an indirect or a direct method (notice the dashed lines in Fig. 7). The difference within the process for the shooting method is depicted in Fig. 12. Similarly, collocation techniques can be used not only in direct methods but also in indirect methods as well, with respect to the Covector Mapping Principle. The reader is urged to consult the works of Huntington et al. [99] for additional information. It should also be noted that the Covector Mapping Principle is satisfied by not only pseudospectral methods but also by Runge-Kutta methods. See [142] as an example in this regard.

When using an indirect approach, the user must compute the quantities of the Hamiltonian function. Unfortunately, this operation requires the user to have at least some knowledge of optimal control theory. Furthermore, even if the user is familiar with the requisite theoretical background, it may be very difficult to construct these expressions for complicated black box applications.

The major drawback for the indirect approach is the robustness. One difficulty is that the user must guess values for the adjoint variables, which is very non-intuitive since they are not physical quantities. Even with a reasonable guess for the adjoint variables, the numerical solution of the adjoint equations can be very ill-conditioned.

As for the direct method, more flexibility can be gained in finding the solution. Since the adjoint equations are not formed explicitly, analytic derivatives are not required. Instead, equivalent information can be computed using sparse finite differences. Consequently, a user with minimal knowledge of optimal control theory can use the method. The method is flexible and new formulations are handled readily. Path inequalities do not require an a priori estimate of the constrained-arc sequence because the active set procedure automatically determines the arc sequence. The method is very robust since the user must guess only the problem variables. Furthermore, the globalization strategy, which is designed to improve a merit function, has a much larger region of convergence than finding a root of the gradient 


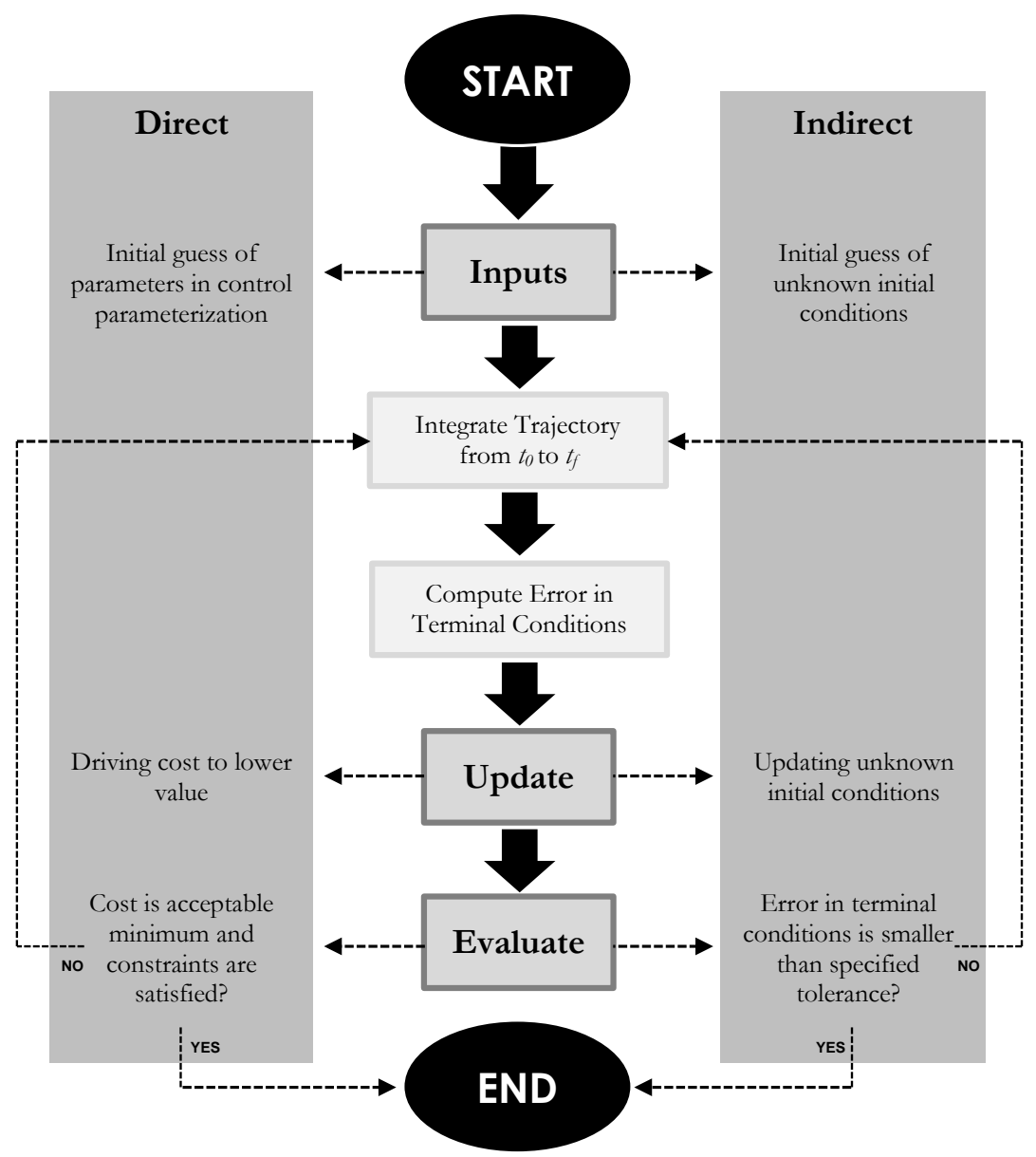

Figure 12: Algorithm for direct and indirect shooting methods

of the Lagrangian, which is the approach used by an indirect method. To sum up, for most applications, the direct method is quite powerful and eliminates the deficiencies of an indirect approach. Indeed, it is often tempting to use a direct method simply because it is more easily implemented and understood. Nevertheless, there may be some situations that are problematic in direct strategies, such as singular arcs and discontinuous control. Articles dedicated to direct and indirect methods in different space missions are tabulated in Table 6 .

The approach is the most important element of the four steps mentioned in the main process of solving a spacecraft trajectory optimization problem. 


\begin{tabular}{|c|c|c|}
\hline Mission & Direct method & Indirect method \\
\hline Typical & $\begin{array}{c}{[143][144][145][41][126]} \\
{[48][105][141][109][64]}\end{array}$ & [99] [69] [146] [147] [148] [79] [149] \\
\hline Interplanetary & $\begin{array}{c}{[150][151][47][152][101]} \\
{[71][45][96][107][153][154]}\end{array}$ & {$[70][51][100][62][63][65]$} \\
\hline Libration & [59] [155] [57] [156] & [89] [157] [158] [159] \\
\hline Rendezvous & {$[160][161]$} & [75] [67] [162] [77] [163] [164] \\
\hline Other & [137] [139] [98] [165] [166] & {$[19]$} \\
\hline
\end{tabular}

Table 6: Numerical approaches in spacecraft trajectory optimization

The choice between the approaches vastly depends on what type of problem is being faced and what solution is actually expected. One good example which shows this dependency is the concept of on-line and off-line implementation of an approach [167]. The first kind of concept assigns maximum priority to the speed of convergence, maybe to the detriment of the accuracy or optimality itself. An example is provided by rendezvous with a moving target or docking maneuvers in which a real-time calculation of the approaching trajectory is often required [160]. As for the latter category, alternatively, the time for convergence is not as important as the optimality of the solution. An example is the long-time scheduling of a space mission involving multiple fly-bys [168].

\section{Solution}

The final step of the spacecraft trajectory optimization process consists of achieving the solution based on the approach developed in the previous section, either analytical or numerical. In Fig. 13, a hierarchy of algorithms used in the literature for spacecraft trajectory optimization is presented which will be used as a scheme in the rest of the section.

If the analytical approach is developed in the previous step, the exact solution will be achieved. In the case of simple spacecraft trajectory optimization problems and sometimes very specific space missions, researchers usually use exact methods. Once again, the simplest example is solving the problem of impulsive orbit which arises with Hohmann transfer approach [15]. This analytical approach clearly ends in an exact solution directly. When a problem is solved analytically, it usually does not involve any iteration. There is no 


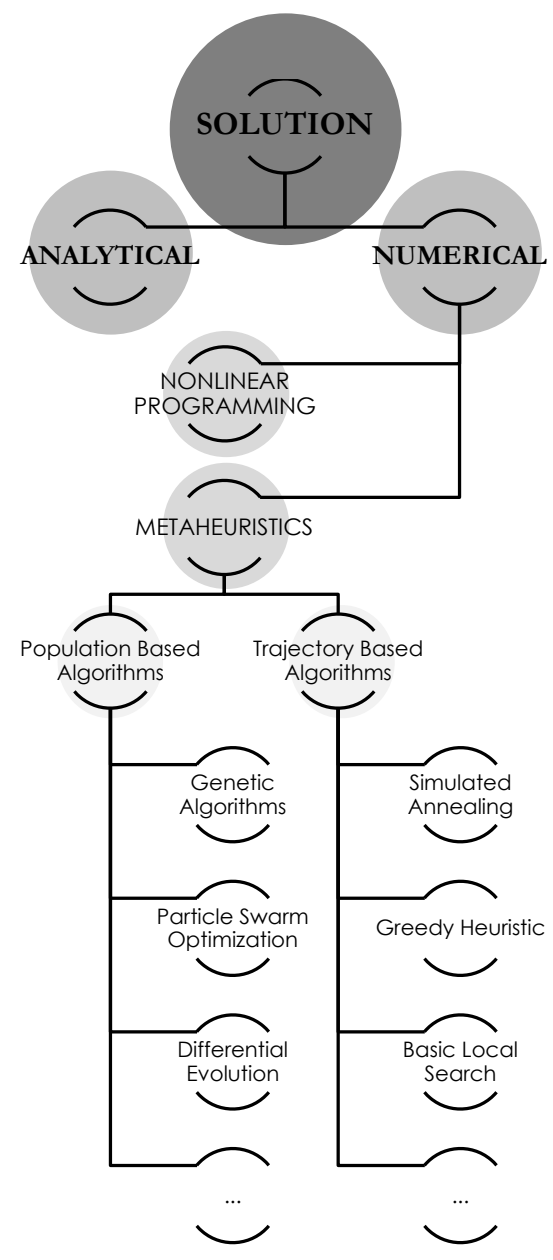

Figure 13: Taxonomy of solutions in spacecraft trajectory optimization

need to use any special numerical technique or iterative procedure to achieve the solution if the approach is truly analytical. As mentioned in the previous section, such cases are very rare in spacecraft trajectory optimization. Some of them are provided in Table 7.

On the contrary, when spacecraft trajectory optimization problems become too complex for exact methods, numerical algorithms, heuristics and, in particular, metaheuristics are often used. In such cases, the problem is considered as a black-box which can be tackled with computational techniques and algorithms. Moreover, the problem imposes less restrictions to the application of different algorithms in this way. 


\begin{tabular}{|c|c|c|c|}
\hline Mission & Author(s) & Reference & Description \\
\hline Typical transfer & da Silva et al. & {$[123]$} & $\begin{array}{l}\text { A complete first-order analytical solution, } \\
\text { which includes the short periodic terms } \\
\text { in an inverse-square force field, between } \\
\text { coplanar orbits with small eccentricities is } \\
\text { presented. }\end{array}$ \\
\hline Interplanetary & Quarta and Mengali & {$[116]$} & $\begin{array}{l}\text { A semi-analytical method is proposed for } \\
\text { the analysis of minimum-time solar sail } \\
\text { heliocentric orbit raising. A closed-form } \\
\text { estimation of the minimum flight time } \\
\text { is presented under the assumption that } \\
\text { the sail characteristic acceleration is suf- } \\
\text { ficiently small. }\end{array}$ \\
\hline Typical transfer & Mengali and Quarta & {$[169]$} & $\begin{array}{l}\text { An analytical approach is developed for } \\
\text { the classical problem of escape from ellip- } \\
\text { tical orbit using a constant radial thrust. } \\
\text { The method is based on graphical rep- } \\
\text { resentations, dedicated to the spacecraft } \\
\text { equipped with low-thrust propulsion sys- } \\
\text { tems. }\end{array}$ \\
\hline Rendezvous & Yang and Cao & {$[81]$} & $\begin{array}{l}\text { The trajectory optimization problem for } \\
\text { autonomous rendezvous of spacecraft with } \\
\text { limited thrust is tackled. Based on the } \\
\text { Lyapunov stability theory, the rendezvous } \\
\text { problem is transformed into an anasymp- } \\
\text { totic stabilization problem of the switch- } \\
\text { ing system. }\end{array}$ \\
\hline Rendezvous & Ma et al. & {$[84]$} & $\begin{array}{l}\text { An analytical state-feedback controller de- } \\
\text { sign algorithm is presented for trajectory- } \\
\text { tracking of circular-orbit rendezvous, con- } \\
\text { sidering actuator failures and exogenous } \\
\text { disturbances. }\end{array}$ \\
\hline Typical transfer & Roa et al. & {$[66]$} & $\begin{array}{l}\text { A novel fully analytic approach based on } \\
\text { logarithmic spirals for spacecraft trajec- } \\
\text { tory optimization in typical orbit transfer } \\
\text { problems is presented. }\end{array}$ \\
\hline
\end{tabular}

Table 7: Solutions for analytical approaches in spacecraft trajectory optimization

\subsection{Nonlinear Programming}

Of the few types of computational techniques commonly used to solve trajectory optimization problems, gradient based methods such as nonlinear programming seems to be the most popular. Over the past decades, nonlinear programming (NLP) has become an indispensable tool for the optimization of space trajectories. These tools are now applied at research and process development stages, in the design stage, and in the online operation of these processes. More recently, the scope of these applications is being extended to cover more challenging, large-scale tasks. The ability to solve large-scale problems cheaply, even online, is aided by recent breakthroughs 
in NLP. They include the development of modern barrier methods, deeper understanding of line search and trust region strategies to aid global convergence, efficient exploitation of second derivatives in algorithmic development, and the availability of recently developed and widely used NLP codes.

Since NLP uses gradient information, it is often capable of relatively quick convergence and very accurate results (within the accuracy of the discretization). They also have well-defined convergence criteria. This has led to their popularity and the development of many individual software packages. A vast number of NLP solvers has been developed since 1970. Some of them are SNOPT [170], DONLP2 [171], filterSQP [172], rSQP++ [173] and KNITRO [174] which has been developed since the late 1990s until the mid 2000s. Another available package is the function $f \operatorname{mincon}()$ of MATLAB optimization toolbox. Developed in 2009, it is a general, multi-purpose constrained parameter optimizer for small, medium, and large spacecraft trajectory optimization problems [101]. Table 8 shows some of the researches in which the fmincon() function is used as the optimizer for spacecraft trajectory optimization in different space missions regarding direct and indirect approaches. The existence of various NLP solvers has led to a number of implementations

\begin{tabular}{cc}
\hline \hline Mission & References \\
\hline Libration Points & {$[175][158]$} \\
Interplanetary & {$[16][151][101][41][126][107][78][65]$} \\
Reentry and others & {$[176][177][178][179]$} \\
Rendezvous & {$[48][78][82][180][86]$} \\
Typical transfer & {$[41][126][48][141]$} \\
\hline \hline
\end{tabular}

Table 8: Application of MATLAB fmincon() in spacecraft trajectory optimization

and very reliable and efficient software packages for large-scale nonlinear programming. Certainly, one of the most recent and useful platforms for NLP is that of the NEOS Server [181]. This server provides state-of-the-art software free of charge to solve optimization problems. Other platforms such as AIMMS [182], AMPL [183], and GAMS [184] have also made the formulation and solution of optimization accessible to a much wider user base.

These NLP algorithms and associated solvers discussed in this section comprise only a sampling of representative codes, based on Newton-type methods. A complete listing is beyond the scope of this survey and the 
reader is referred to the NEOS software guide [181] for a more complete selection and description of NLP codes. Moreover, important issues such as scaling and numerical implementations to improve precision have not been covered here. Readers are referred to [185] for more information on NLPs.

The most noticeable problem with gradient based methods is that they require an initial guess of all the parameters of the system. Since all nodal state and control values are parameterized, the analyst must have some $a$ priori knowledge of the optimal trajectory. The consequences of poor initial guessing are usually failure to converge or convergence upon a non-global optimum solution in the cost function.

Having the initial guess for the gradient-based method is a challenging issue in spacecraft trajectory optimization. A method to address this issue was first proposed in 1995 [186], which leads to the presentation of metaheuristics, an alternative way to solve the discrete direct or indirect trajectory optimization formulations.

\subsection{Metaheuristics}

In recent years, there have been significant advances in the use of metaheuristics to approximate solutions of spacecraft trajectory optimization problems. A metaheuristic is an iterative master process that guides and modifies the operations of subordinate heuristics to efficiently produce high-quality solutions [187]. It may manipulate a complete (or incomplete) single settlement or a collection of settlements in every iteration. The subordinate heuristics may be high (or low) level procedures, or a simple local search, or just a construction method. The family of metaheuristics includes, but is not limited to, genetic algorithms, particle swarm optimization, simulated annealing, and their hybridizations.

Metaheuristics provide decision-making managers with robust tools that obtain high-quality solutions, in reasonable time horizons, to important applications specifically in aerospace optimization problems. The well-known survey by Blum and Roli [188] divides metaheuristics into two main categories including single solution algorithms and population based algorithms. The first category, also referred to trajectory-based algorithms, gathers local search, greedy heuristic, simulated annealing, tabu search, iterated local search, etc [187]. The second category, which is more practical in spacecraft trajectory optimization, regroups evolutionary algorithms such as genetic algorithms [189], evolution strategies [190], genetic programming [191], particle 
swarm optimization [192], etc. Survey by Xiong [193] also confirms this taxonomy with a slight difference in which the single solution algorithms are separated into two categories called trajectory based and multi-trajectory based algorithms. Other taxonomies exist as well that try to put algorithms in different groups [194], [195].

Among metaheuristics, evolutionary algorithms are particularly suited for most of the spacecraft trajectory optimization problems. A Generic diagram for most of the algorithms in this group is presented in Fig. 14.

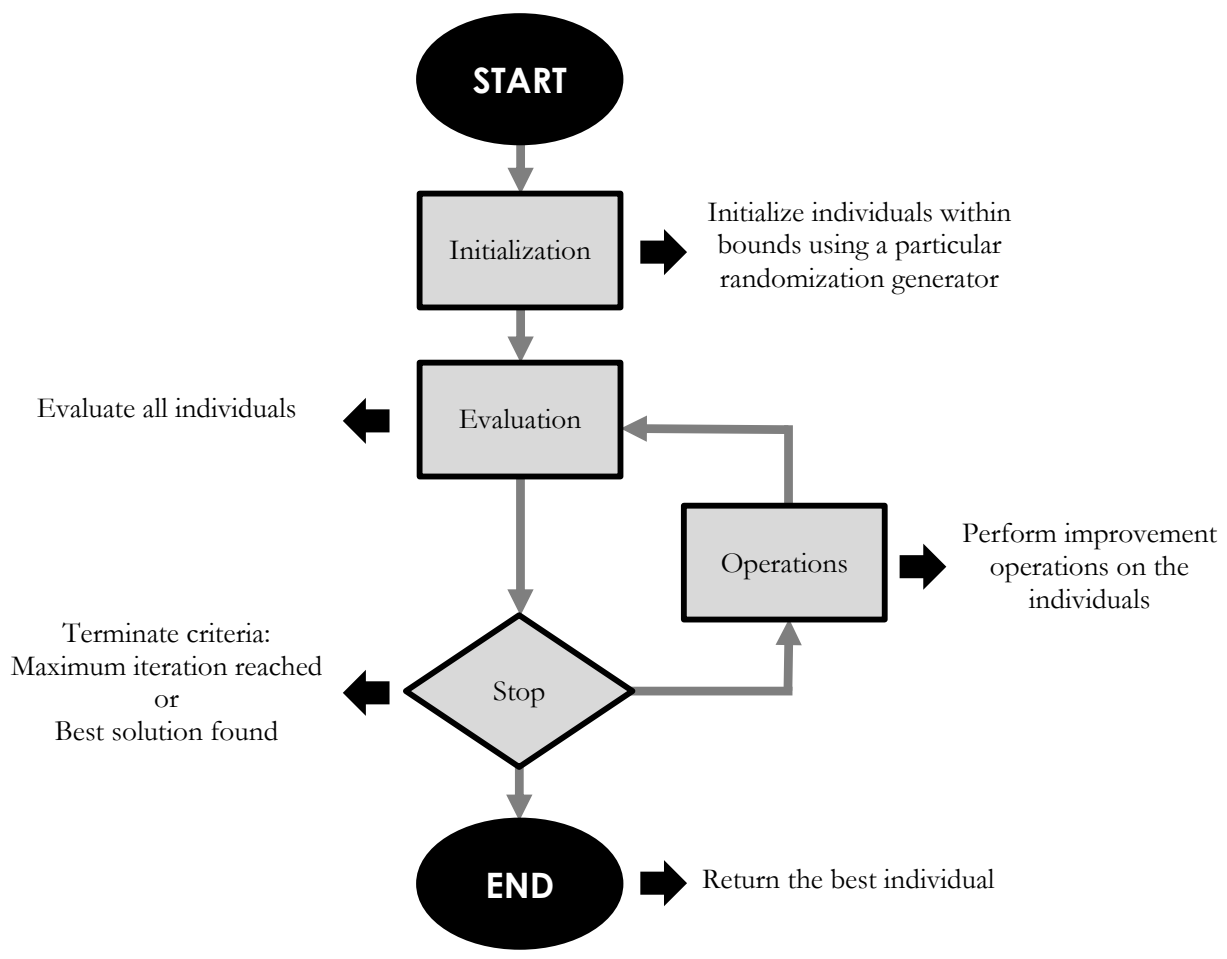

Figure 14: Generic flowchart of evolutionary algorithms

The most recurrent instances in EAs group are Genetic Algorithms (GAs) which have been used in many papers. Besides GAs, Differential Evolution (DE), has also been used frequently in the solution of spacecraft trajectory optimization. In these algorithms, the information about the structure of the problem can be incorporated in order to improve the efficiency of the algorithms.

The performance of different algorithms on different benchmark problems 
are compared in some researches. The tested algorithms in these studies include, besides GA and DE, EAs such as Particle Swarm Optimization (PSO), Ant colony optimization (ACO) and also local search algorithms such as Simulated Annealing (SA). As a summarization, Table 9 contains references of the most used EAs in spacecraft trajectory optimization problems.

\begin{tabular}{|c|c|c|c|c|}
\hline Model & Mission & GA & PSO & $\mathrm{DE}$ \\
\hline \multirow{4}{*}{ Impulsive } & Rendezvous & [196] [197] [19] & [196] [198] & [196] \\
\hline & Typical transfer & {$[22][24][25][26][110][23]$} & {$[24][25][112][199]$} & {$[25]$} \\
\hline & Interplanetary & {$[200][201]$} & {$[202]$} & - \\
\hline & Gravity assist & $\begin{array}{c}{[24][25][30][31][32]} \\
{[34][35][36][196]}\end{array}$ & $\begin{array}{l}{[24][25][31][34][35]} \\
\quad[28][196][203]\end{array}$ & $\begin{array}{c}{[25][31][34][35][33]} \\
{[196][203]}\end{array}$ \\
\hline \multirow{5}{*}{ Continuous } & Rendezvous & {$[204][156][75]$} & {$[86][164]$} & - \\
\hline & Typical transfer & $\begin{array}{c}{[69][114][205][146][147]} \\
{[112]}\end{array}$ & {$[69][146]$} & - \\
\hline & Interplanetary & {$[45][200][107][206][207]$} & [61] [208] [62] [112] & - \\
\hline & Libration points & [209] [210] [85] & [58] [112] & [87] [72] [211] \\
\hline & Others & [106] [98] & {$[165]$} & - \\
\hline
\end{tabular}

Table 9: Evolutionary algorithms in spacecraft trajectory optimization

It can be highlighted that GAs are the first choice for most of the spacecraft trajectory optimization problems, perhaps due to their availability and ease of use. However, it is difficult to know the particular variant used (codification, operators, etc.) from the contents of the papers. Another observation regarding the literature confirms the fact that metaheuristics are more used in problems based on impulsive models rather than continuous models. To be more specific, gravity assist missions are the problems which are tackled mostly by evolutionary algorithms. Table 10 provides some of the research in which the other metaheuristics instead of GA, PSO and DE, are employed in spacecraft trajectory optimization.

According to the literature, researchers tend to use EAs more than trajectorybased algorithms. However, the EAs have drawbacks which, to some authors, make it unacceptable as a primary means of trajectory optimization [1]. Firstly, as the algorithm is probabilistic, the difference in the output solution in different runs can be high. Second, there are no well-defined convergence criteria (i.e., no necessary and sufficient conditions), such as those used in NLP algorithms. 


\begin{tabular}{cccc}
\hline \hline Model & Mission & Metaheuristic & Reference \\
\hline Impulsive & Rendezvous & Simulated Annealing & {$[19]$} \\
Impulsive & Gravity assist & Multiple Trajectory Search & {$[24]$} \\
Impulsive & Typical transfer & Monotonic Basin Hopping & {$[25]$} \\
Impulsive & Gravity assist & A Novel Global Optimization Algorithm & {$[29]$} \\
Impulsive & Gravity assist & Improved Ant Colony Optimization & {$[32]$} \\
Impulsive & Gravity assist & Basic Best Start Algorithm & {$[34]$} \\
Continuous & Typical transfer & Imperialist Competitive Algorithm & {$[69]$} \\
Continuous & Interplanetary & Improved Imperialist Competitive Algorithm & {$[96]$} \\
Continuous & Reentry & A Pigeon Inspired Algorithm & {$[97]$} \\
Continuous & Rendezvous & Interval Algorithm & {$[156]$} \\
Impulsive & Rendezvous & Simulated Annealing & {$[197]$} \\
Impulsive & Interplanetary & Monotonic Basin Hopping & {$[206]$} \\
Continuous & Rendezvous & Simulated Annealing & {$[212]$} \\
Continuous & Typical transfer & Imperialist Competitive Algorithm & {$[213]$} \\
Continuous & Typical transfer & Estimation of Distribution Algorithms & {$[214]$} \\
Continuous & Interplaanetary & Evolutionary Neurocontrol & {$[215]$} \\
\hline \hline
\end{tabular}

Table 10: Other metaheuristics in spacecraft trajectory optimization

It should also be highlighted that metaheuristics has been used not only for spacecraft trajectory optimization, but also for space orbit design problems as well. A typical example is the use of EAs for minimizing the average revisit time of a space mission over a particular target site during the specified days [216].

\subsection{Hybrid algorithms}

In recent years, many works have relied on cooperative (or hybrid) optimization techniques. In many cases, the best results are obtained with this kind of techniques, especially in real-life problems. At the beginning, cooperation was mainly carried out between several metaheuristics. But nowadays, more and more cooperation schemes between metaheuristics and exact approaches are proposed. These strategies usually give good results because they are able to exploit simultaneously the advantages of several types of algorithms or methods. For example, it may allow giving quality guarantees to the identified solutions or may reduce the sensitivity to the initial guess.

The first attempt, in 1995 [186], was intended to be a solution of the multi-point boundary value problem (MPBVP) for systems with Mayer cost functions, and linearly appearing controls. The final numerical solution of the optimality conditions was initialized using guesses for the switching times, 


\begin{tabular}{|c|c|c|c|}
\hline Space Mission & Author(s) & Reference & Description \\
\hline Rendezvous & Luo et al. & [17] & $\begin{array}{l}\text { A two step hybrid algorithm based on GA } \\
\text { and SQP is proposed. }\end{array}$ \\
\hline Typical transfer & Shafieenejad et al. & [69] & $\begin{array}{l}\text { A GA-PSO hybrid algorithm is employed } \\
\text { in a low-thrust transfer within an indirect } \\
\text { method. }\end{array}$ \\
\hline Gravity assist & Vasile and Zuiani & {$[24]$} & $\begin{array}{l}\text { A hybrid algorithm called MACS is pro- } \\
\text { posed based on a number of heuristics to } \\
\text { combine local and global search. The al- } \\
\text { gorithm outperforms NSGA II and other } \\
\text { metaheuristics in different space missions. }\end{array}$ \\
\hline Gravity assist & Izzo et al. & {$[69]$} & $\begin{array}{l}\text { A technique called GASP is proposed } \\
\text { which creates intelligent initial individuals } \\
\text { according to multi gravity assist mission } \\
\text { when hybridized with other metaheuris- } \\
\text { tics such as GA, PSO and DE. }\end{array}$ \\
\hline Rendezvous & Luo et al. & {$[18]$} & $\begin{array}{l}\text { A two step algorithm called GASM is pro- } \\
\text { posed in which the GA serves a starter } \\
\text { engine and the SM (Simplex Method) can } \\
\text { then refine to pinpoint the minimum pa- } \\
\text { rameter solution with fine resolution. }\end{array}$ \\
\hline Gravity assist & Vasile and Locatelli & {$[34]$} & $\begin{array}{l}\text { A hybrid algorithm is proposed which } \\
\text { is based on domain decomposition and } \\
\text { stochastic multiagent search technique. }\end{array}$ \\
\hline Gravity assist & Englander et al. & {$[35]$} & $\begin{array}{l}\text { A hybrid algorithm based on GA, PSO } \\
\text { and DE is proposed for mission plan- } \\
\text { ning. In this combinatorial-continuous ap- } \\
\text { proach, GA is used to find the optimal se- } \\
\text { quence of planets while cooperative PSO- } \\
\text { DE is used for trajectory optimization of } \\
\text { the selected sequence of planets. }\end{array}$ \\
\hline Gravity assist & Vasile et al. & [33] & $\begin{array}{l}\text { A robust algorithm based on a hybridiza- } \\
\text { tion of DE and the logic behind MBH } \\
\text { (Monotonic Basin Hopping) is proposed. } \\
\text { The algorithm outperforms DE on some } \\
\text { difficult space trajectory design problems. }\end{array}$ \\
\hline Interplanetary & Vavrina and Howell & {$[150]$} & $\begin{array}{l}\text { An algorithm is developed through the } \\
\text { hybridization of GA and GALLOP (a } \\
\text { gradient-based direct method). }\end{array}$ \\
\hline Rendezvous & Sentinella and Casalino & [196] & $\begin{array}{l}\text { A hybrid evolutionary algorithm which } \\
\text { synergistically exploits DE, GA and PSO, } \\
\text { has been developed. The cooperative pro- } \\
\text { cedure runs the three basic algorithms in } \\
\text { parallel, while letting the best individuals } \\
\text { migrate to the other populations at pre- } \\
\text { scribed intervals. }\end{array}$ \\
\hline Typical transfer & Shirazi & [217] & $\begin{array}{l}\text { A hybrid metaheuristic based on GA and } \\
\text { SA is employed. The algorithm outper- } \\
\text { forms typical GA and SA. }\end{array}$ \\
\hline Libration points & Lei et al. & {$[88]$} & $\begin{array}{l}\text { A hybridization of PSO with DE is devel- } \\
\text { oped. The algorithm has advantages over } \\
\text { basic algorithms which suffer from slow } \\
\text { convergence and loosing exploitation ca- } \\
\text { pability. }\end{array}$ \\
\hline Rendezvous & Ma and Xu & [156] & $\begin{array}{l}\text { An interval algorithm (IA) combining } \\
\text { the gradient-based optimization method is } \\
\text { presented for space rendezvous problem. }\end{array}$ \\
\hline
\end{tabular}

Table 11: Hybrid algorithms in spacecraft trajectory optimization 
generated by examining the results of an NLP shooting technique initialized, in turn, by a shooting technique incorporated with GA. The solved problem was the reorientation of an inertially symmetric spacecraft. Other authors, such as those of [218], have opted to concentrate on modifying the components of the GA to improve the performance. Though the goal of that work was to improve the GA components themselves, the framework is relevant because it allows a more general representation of the control history in a direct shooting technique with a GA while using the result to initialize the NLP solution of a direct collocation method.

In the literature, for example [33] and [24], it is also demonstrated that a hybridization between global optimization techniques, generally applicable to black-box problems, with ad hoc branch-and-prune methods and exploiting the properties of the problem (e.g., continuity and differentiability, periodicity, symmetry, modularity) can greatly improve convergence, specifically when applied to spacecraft trajectory design. Hybridization may also refer to switching between global and local minimizers in an optimization algorithm in some researches [24], [29], [34]. However, it does not only refer to the algorithms. It sometimes refers to the approaches. For example, taking advantage of both direct and indirect approach features in solving a trajectory optimization problem in a space mission is sometimes called a hybrid technique [113], [50]. Some examples regarding the hybrid algorithms applied in spacecraft trajectory optimization are provided in Table 11.

Analyses regarding the effectiveness of the hybrid algorithms are encouraging as, for the same computational effort (measured in number of function evaluations), hybrid algorithms were converging more accurately than common algorithms in many cases.

\section{Summary and discussion}

This review tried to provide a complete taxonomy of concepts within spacecraft trajectory optimization. This section provides the overall discussion regarding spacecraft trajectory optimization based on the literature referred to in this review along with some issues in this field. Moreover, future trends and useful considerations regarding the upcoming ideas in spacecraft trajectory optimization are put forward. 


\subsection{Highlights}

By having an overview of the literature, several points can be highlighted. They include the relation between direct and indirect methods, metaheuristics and computational issues in spacecraft trajectory optimization which will be discussed briefly in this subsection.

The two branches of direct and indirect methods play an important role in categorizing the approaches. These two different philosophies have led to a dichotomy in the space community. Researchers who focus on indirect methods are principally interested in differential equation theory, while researchers who focus on direct methods are more interested in optimization techniques. While seemingly unrelated, there are a lot of common characteristics between these two methods. Specifically, as discussed in this review, in recent years researchers have delved quite deeply into the connections between the direct and indirect methods. They have discovered that the optimality conditions from many direct methods have a well-defined meaningful relationship. Thus, these two classes of methods are merging as time goes by. Covector Mapping Principle is actually the connectivity that fills the gap between these two branches [11]. It renders the terms direct and indirect obsolete in the modern viewpoint of spacecraft trajectory optimization and reveals that the obvious and shorter path of dualization first and computing afterward is strewn with difficulties while a longer path of reversing the operations eliminates the curse of sensitivity.

Regarding metaheursitics, the key point is selecting an algorithm intelligently for spacecraft trajectory optimization. The question remains unanswered about which algorithm is appropriate for a specific class of trajectory optimization problems. One reason is the definition of a good algorithm, which is different from paper to paper. Ideally, a good algorithm is capable of solving most of the instances of a given spacecraft trajectory optimization problem faster than a bad algorithm regardless of the method (direct or indirect) in an acceptable range of accuracy. Typically computer time is used to measure algorithm speed; however, when this is done it is imperative that all testing be done using the same hardware, compiler options, and operating system. The number of function evaluations can be used instead of (or in addition to) computer time, but then one must carefully define a function evaluation. Furthermore, in order to make a fair comparison between algorithms, it is important to consider several factors. These factors include testing a large suite of instances of the problem, using the same initial guess, and comparing them based on the same convergence criteria. When com- 
paring one metaheuristic to another in a spacecraft trajectory optimization problem, it is common to perform benchmark tests using a suite of standard problems.

Four important computational issues that arise in the numerical approaches in spacecraft trajectory optimization are (i) consistent approximations for the solution of differential equations, (ii) the scaling of optimal control problem (iii), exploitation of sparsity in the problem, and (iv) computation of derivatives of the objective and constraint functions. The manner in which the differential equations are discretized is of great important because an inconsistent approximation to the differential equations can lead to either non-convergence or convergence of the optimization algorithm to the infeasible solution. Scaling and exploitation of sparsity in the problem are issues that greatly affect both computational efficiency and convergence of the algorithm. Finally, the manner in which derivatives are computed is also of great importance because accurate derivatives can greatly improve both computational efficiency and reliability.

\subsection{Issues}

During the last few decades, every year several papers regarding an innovative concept, approach or method for spacecraft trajectory optimization is published. The majority of the publications present truly novel ideas in this field. However, sometimes re-iteration of existing knowledge in this subject is introduced as a novelty. One example is the application of metaheuristics, more specifically EAs, in spacecraft trajectory optimization. Although some novelties are perfectly introduced in many publications, some papers are dedicated to the application of existing algorithms or techniques in specific space missions. The other similar issue is the parameter tuning of algorithms, which is called a novelty sometimes. Unfortunately, some of them do not represent a real advance in the field.

Another suggestion for improving the quality of the literature in this field is standardization of the whole evaluation process of the algorithms. It means using a high number of instances for evaluation of the proposed trajectory optimization algorithms or methods in articles. When performing empirical experiments with methods in spacecraft trajectory optimization, the goal is to show that a specific method performs better than other methods on a class of space mission instances with respect to some predefined objective, which is computational time and $\backslash$ or solution quality. However, an overview of the published papers in this field confirms that experiments are performed only 
on a few instances or even on a single instance, instead of using a class of space mission instances or randomly sampled orbits.

The next issue is the comparison of the results with other methods. The best way to show that a new method is really successful is to demonstrate that it outperforms state-of-the-art approaches. In some of the publications cited in this review, it is not clear whether a state-of-the-art method has been used for the comparisons or not. An alternative to show that the new method is at least competitive or interesting is to demonstrate that it outperforms some standard approaches. Unfortunately in most of the publications the proposed method is only compared to a few other approaches. Even more dramatic is the fact that in some papers no comparisons are performed at all.

There are some other issues as well, which are not discussed in detail here. For example, the experimental conditions are not completely clear in most of the publications, sometimes best solutions are used for comparisons instead of average solutions, and statistical tests are not performed in most of the publications.

\subsection{Suggestions for future trends}

Several research lines can be identified as relevant subjects for future works which take the field forward in promising directions. This section is not by any means meant as a catalogue of or a roadmap for excellent research in spacecraft trajectory optimization, its only purpose is to point out some properties that the authors consider to be good research suggestions and practices, and some promising areas in which a lot of research is still needed.

As the first suggestion, any research on spacecraft trajectory optimization should be adequately framed in the general literature. Adequately framing a method entails deconstructing it, showing which components it consists of, and how these components were adapted to the specific space mission that is being solved. For this to be at all possible, it is promising to explain new spacecraft trajectory optimization approaches using the general optimization terminology as introduced in this review. Clarifying the four key items including model, objective, approach and the procedure for achieving the solution can help the readers understand the structure of the problem. This leads to deconstruction of the problem and reproducibility.

Following the proper terminology, all trajectory design should return to a situation in which methods are developed based on insight into the structure of the problem. Especially, research in spacecraft trajectory optimization 
should be applauded if it yields insight into the reasons why specific algorithms, methods or techniques work well on specific space missions. For example, the application of global optimization algorithms such as GA, PSO, etc. to space trajectory problems often considers the problem as a black box with limited exploitation of problem characteristics. However, in the component-based view of spacecraft trajectory optimization, concepts from one or a set of different frameworks can be combined into ever more powerful approaches and algorithms, such as hybrid approaches and algorithms, which are discussed previously. Such concepts can exploit problem characteristics, providing sensible improvements over the direct application of general purpose methods. The result of such a process is a deep insight into which components are responsible for the core optimization power of the overall method. Potentially, such analyses allow the spacecraft trajectory designer to draw important conclusions on why the method works as well as it does, by proving a relationship between the properties of the optimization method and the structure of the space mission problem that is being solved. Selfadaptive methods, specifically metaheuristics that automatically tune their parameters according to the dynamic stiffness of spacecraft trajectory optimization problem, also present an interesting line of future research.

Rather than spacecraft trajectory optimization, space mission design is also a challenging issue which is totally connected with spacecraft trajectory optimization itself. This process, better known as space mission planning, refers to two sequential steps; design the space mission and then optimizing the trajectory related to the mission. Many space mission planning problems are constructed in such a way that they include both real-valued variables and categorical variables. The categorical variables will typically specify the sequence of events that qualitatively describe the trajectory or mission, and the real-valued variables will represent the launch date, flight times between planets, magnitudes and directions of rocket burns, flyby altitudes, etc., [35]. For example in multi gravity assist maneuvers, the designer must choose both a set of discrete categorical variables defining the sequence of gravity assists, or flybys, to be performed and a set of real-valued variables that define the trajectory corresponding to that sequence of flybys. The categorical variables represent the planets chosen for gravity assists, and the real-valued variables represent other parameters relevant to the trajectory, such as the date of launch and the flight times between each planet in the sequence. Therefore, the mission planning can thus be considered as two nested optimization problems including an inner loop that optimizes the trajectory for 
a given mission sequence, and an outer-loop scheduling problem that chooses the optimal sequence of gravity assists. Such concepts involve using EAs in both combinatorial and continuous domain, which is an interesting topic for future researches, since little research is dedicated to them.

Another interesting subject is that of scalarization techniques in spacecraft trajectory optimization, which has not received much attention in the literature. Multi-objective trajectory optimization methods utilize various scalarization functions in different researches depending on the space mission, approach, type and number of minor cost functions. In most scalarization functions, preference information of the decision maker is taken into consideration. After the scalarization phase, the widely developed theory and methods of single objective optimization can be used to deal with the problem. However, no current research can be found regarding the comparison of different scalarization techniques in spacecraft trajectory optimization, or at least in a specific space mission.

The other new trend is designing global optimization metaheuristics which are useful in automatically finding and selecting good trajectory options between the many often possibilities one has in the preliminary phases of mission design. Their use and efficiency are established for chemical propulsion problems of high complexity (i.e., large launch windows and multiple flybys) whenever approaches more sophisticated than the straightforward use of standard algorithms are adopted. Preliminary results in this sense are already available and point to an increased need for computational resources. It seems likely that future research results will aim at proving the use of these techniques for the automated computation of low-thrust trajectories as well.

Comparing different trajectory optimization approaches has so far been a largely unstructured affair, with testing procedures being determined on the fly and sometimes with a specific outcome in mind. Although some authors have developed procedures to make a statistically sound comparison, widespread acceptance of such procedures is lacking. Perhaps a set of tools is needed, i.e., a collection of optimized trajectories or libraries specifically designed to determine the relative quality of a set of methods on a set of problem instances. These should both be easy to use, and their results should be easy to interpret. Until such tools are available and a specific comparison protocol is enforced by journal editors and reviewers, the door is left open for researchers to select the method of comparison that proves the point it is intended to prove. Moreover, such contributions can be published even if they do not contain any novel method or a method that outperforms all 
existing approaches.

\section{Conclusion}

A review for solving spacecraft trajectory optimization problems has been given in this article. The solving process is decomposed into four key steps of mathematical modeling of the problem, defining the objective functions, development of an approach and obtaining the solution of the problem. Using these steps, several subcategories for each step have been described. Subsequently, important classifications and their characteristics have been discussed for solving spacecraft trajectory optimization problems. Finally, a brief discussion has been given on how to decide and choose in each step.

This review is considered complementary to most of the previously published survey articles on spacecraft trajectory optimization. It reflects most of the research and efforts that has been carried out over the past decade while simultaneously providing a summary of the vast amount of work that was done up to this point. The material in this review has been presented to give the reader an understanding of how methods, techniques and algorithms are categorized for spacecraft trajectory optimization problems. It is also worth noting that a great deal of discussion has been given to the distinction between different categories, not just in this review but also in previously published surveys.

To sum up, trajectory design and optimization has a broad variety of applications in different fields, particularly in aerospace engineering. The solution of a trajectory optimization problem that minimizes a cost function subject to nonlinear differential equations of motion and various types of constraints may be obtained by either an analytical approach or a numerical approach. From the viewpoint of numerical computation, spacecraft trajectory optimization is a hard global optimization problem, which is even more difficult when the analytical expressions of the objective function or the constraints are not usually available. Moreover, even simple bi-dimensional cases display an enormous number of local optima. All of the iterative techniques and algorithms for spacecraft trajectory optimization can present convergence difficulties (non-convergence, slow convergence, etc.). These difficulties should be considered for each specific problem since a general technique for all spacecraft trajectory optimization problems does not exist. It does not make sense to ask general questions such as "Are direct methods better than indirect methods? "or "Is Genetic Algorithm better than Particle 
Swarm Optimization in spacecraft trajectory design?". The answer to such questions can only be "It depends on the space mission and the mission requirements". This is not to say that all approaches, methods and algorithms are equally powerful, nor that it is impossible to obtain meaningful insight into whether a specific method is more suitable for solving a specific class of trajectory optimization problems than another. Viewing spacecraft trajectory optimization concepts as sets of general ideas allows a broader view of the literature and allows for the discovery of similarities between the structure and inner workings of methods that remain opaque if only the label the author of the method has chosen for it is considered. This is certainly true in the modern view of spacecraft trajectory optimization, in which ideas may combine concepts from different frameworks and the framework that is used to name the method is a matter of the author's personal opinion. Choosing a method for solving the spacecraft trajectory optimization problem is based largely on the type of problem to be solved and the amount of time that can be invested in coding. Various extensions to the currently employed approaches offer opportunities and challenges for future works.

\section{Acknowledgments}

This research is supported by La Caixa Fellowship, the Basque Government through the BERC 2014-2017, the Research Groups 2013-2018 (IT609-13) and Elkartek programs and by Spanish Ministry of Economy and Competitiveness MINECO: BCAM Severo Ochoa excellence accreditation SEV-2013-0323, TIN2016-78365R and TIN2017-82626R projects.

\section{References}

[1] J. T. Betts, Survey of numerical methods for trajectory optimization, Journal of Guidance, Control, and Dynamics 21 (2) (1998) 193-207.

[2] B. A. Conway, A survey of methods available for the numerical optimization of continuous dynamic systems, Journal of Optimization Theory and Applications 152 (2) (2012) 271-306.

[3] P. A. de Sousa-Silva, M. O. Terra, A survey of different classes of earthto-moon trajectories in the patched three-body approach, Acta Astronautica 123 (2016) 340 - 349, special Section: Selected Papers from 
the International Workshop on Satellite Constellations and Formation Flying 2015.

[4] Y. Luo, J. Zhang, G. Tang, Survey of orbital dynamics and control of space rendezvous, Chinese Journal of Aeronautics 27 (1) (2014) 1-11.

[5] S. N. D'Souza, N. Sarigul-Klijn, Survey of planetary entry guidance algorithms, Progress in Aerospace Sciences 68 (2014) 64-74.

[6] M. Shirobokov, S. Trofimov, M. Ovchinnikov, Survey of station-keeping techniques for libration point orbits, Journal of Guidance, Control, and Dynamics 40 (5) (2017) 1085-1105.

[7] M. Xu, Y. Liang, K. Ren, Survey on advances in orbital dynamics and control for libration point orbits, Progress in Aerospace Sciences 82 (2016) 24-35.

[8] G. Huang, Y. Lu, Y. Nan, A survey of numerical algorithms for trajectory optimization of flight vehicles, Science China Technological Sciences 55 (9) (2012) 2538-2560.

[9] J. T. Betts, Practical methods for optimal control and estimation using nonlinear programming, SIAM, 2010.

[10] B. A. Conway, Spacecraft trajectory optimization, Vol. 29, Cambridge University Press, 2010.

[11] I. M. Ross, A primer on Pontryagin's principle in optimal control, 2nd Edition, Collegiate publishers, 2015.

[12] D. E. Kirk, Optimal control theory: an introduction, Courier Corporation, 2012.

[13] H. D. Curtis, Orbital mechanics for engineering students, ButterworthHeinemann, 2014.

[14] J. Sims, S. Flanagan, Preliminary design of low-thrust interplanetary missions, No. AAS 99-338, AAS/AIAA Astrodynamics Specialist Conference.

[15] W. E. Wiesel (Ed.), Space Flight Dynamics, CreateSpace Independent Publishing Platform, 2010. 
[16] X. Y. Jiang, Y. J. Lian, H. B. Zhang, G. J. Tang, A multi-impulse extended method for low-thrust trajectory optimization, in: 2013 6th International Conference on Recent Advances in Space Technologies (RAST), 2013, pp. 365-368.

[17] Y.-Z. Luo, G.-J. Tang, Z.-G. Wang, H.-Y. Li, Optimization of perturbed and constrained fuel-optimal impulsive rendezvous using a hybrid approach, Engineering Optimization 38 (8) (2006) 959-973.

[18] Y.-Z. Luo, G.-J. Tang, H. yang Li, Optimization of multiple-impulse minimum-time rendezvous with impulse constraints using a hybrid genetic algorithm, Aerospace Science and Technology 10 (6) (2006) 534540 .

[19] Y.-Z. Luo, J. Zhang, H. yang Li, G.-J. Tang, Interactive optimization approach for optimal impulsive rendezvous using primer vector and evolutionary algorithms, Acta Astronautica 67 (3-4) (2010) 396-405.

[20] H.-Y. Li, Y.-Z. Luo, Jin-Zhang, G.-J. Tang, Optimal multi-objective linearized impulsive rendezvous under uncertainty, Acta Astronautica 66 (3-4) (2010) 439-445.

[21] Y.-Z. Luo, G.-J. Tang, Y.-J. Lei, Optimal multi-objective linearized impulsive rendezvous, Journal of Guidance, Control, and Dynamics 30 (2) (2007) 383-389.

[22] O. Abdelkhalik, D. Mortari, N-impulse orbit transfer using genetic algorithms, Journal of Spacecraft and Rockets 44 (2) (2007) 456-460.

[23] O. Abdelkhalik, A. Gad, Optimization of space orbits design for earth orbiting missions, Acta Astronautica 68 (7-8) (2011) 1307-1317.

[24] M. Vasile, F. Zuiani, Multi-agent collaborative search: an agent-based memetic multi-objective optimization algorithm applied to space trajectory design, Proceedings of the Institution of Mechanical Engineers, Part G: Journal of Aerospace Engineering 225 (11) (2011) 1211-1227.

[25] M. Vasile, E. Minisci, M. Locatelli, Analysis of some global optimization algorithms for space trajectory design, Journal of Spacecraft and Rockets 47 (2) (2010) 334-344. 
[26] P. L. Myers, D. B. Spencer, Application of a multi-objective evolutionary algorithm to the spacecraft stationkeeping problem, Acta Astronautica 127 (2016) 76-86.

[27] L. Mingtao, Z. Jianhua, Impulsive lunar halo transfers using the stable manifolds and lunar flybys, Acta Astronautica 66 (9-10) (2010) 14811492 .

[28] C. Yang, B. He-xi, L. Jun-feng, Trajectory analysis and design for a jupiter exploration mission, Chinese Astronomy and Astrophysics 37 (1) (2013) 77-89.

[29] B. Addis, A. Cassioli, M. Locatelli, F. Schoen, A global optimization method for the design of space trajectories, Computational Optimization and Applications 48 (3) (2011) 635-652.

[30] F. A. Zotes, M. S. Penas, Delta-v genetic optimisation of a trajectory from earth to saturn with fly-by in mars, in: IEEE Congress on Evolutionary Computation, 2010, pp. 1-6.

[31] D. Izzo, V. M. Becerra, D. R. Myatt, S. J. Nasuto, J. M. Bishop, Search space pruning and global optimisation of multiple gravity assist spacecraft trajectories, Journal of Global Optimization 38 (2) (2006) $283-296$.

[32] M. Ceriotti, M. Vasile, MGA trajectory planning with an ACO-inspired algorithm, Acta Astronautica 67 (9-10) (2010) 1202-1217.

[33] M. Vasile, E. Minisci, M. Locatelli, An inflationary differential evolution algorithm for space trajectory optimization, IEEE Transactions on Evolutionary Computation 15 (2) (2011) 267-281.

[34] M. Vasile, M. Locatelli, A hybrid multiagent approach for global trajectory optimization, Journal of Global Optimization 44 (4) (2009) 461-479.

[35] J. A. Englander, B. A. Conway, T. Williams, Automated mission planning via evolutionary algorithms, Journal of Guidance, Control, and Dynamics 35 (6) (2012) 1878-1887. 
[36] O. Abdelkhalik, A. Gad, Dynamic-size multiple populations genetic algorithm for multigravity-assist trajectory optimization, Journal of Guidance, Control, and Dynamics 35 (2) (2012) 520-529.

[37] G. Lantoine, Methodology for robust optimization of low-thrust trajectories in multi-body environments, Ph.D. thesis, Georgia Institute of Technology (2010).

[38] K. Sankaran, E. Y. Choueiri, S. C. Jardin, Comparison of simulated magnetoplasmadynamic thruster flowfields to experimental measurements, Journal of Propulsion and Power 21 (1) (2005) 129-138.

[39] J. Dankanich, Low-thrust mission design and application, in: 46th AIAA/ASME/SAE/ASEE Joint Propulsion Conference \& Exhibit, American Institute of Aeronautics and Astronautics, 2010, pp. 1-19.

[40] I. D. Boyd, Numerical modeling of spacecraft electric propulsion thrusters, Progress in Aerospace Sciences 41 (8) (2005) 669-687.

[41] E. Taheri, Rapid space trajectory generation using a Fourier series shape-based approach, Michigan Technological University, 2014.

[42] V. V. Ivashkin, I. V. Krylov, Optimization of transfer trajectories to the apophis asteroid for spacecraft with high and low thrust, Cosmic Research 52 (2) (2014) 106-117.

[43] P. Gurfil, Modern astrodynamics, Vol. 1, Butterworth-Heinemann, 2006.

[44] D. A. Vallado, Fundamentals of Astrodynamics and Applications, 4th Ed., Microcosm Press, 2013.

[45] S. Li, Y. Zhu, Y. Wang, Rapid design and optimization of low-thrust rendezvous/interception trajectory for asteroid deflection missions, Advances in Space Research 53 (4) (2014) 696-707.

[46] J. L. Junkins, A. B. Younes, R. M. Woollands, X. Bai, Picard iteration, chebyshev polynomials and chebyshev-picard methods: Application in astrodynamics, The Journal of the Astronautical Sciences $60(3-4)(2013) 623-653$. 
[47] D. J. Gondelach, R. Noomen, Hodographic-shaping method for lowthrust interplanetary trajectory design, Journal of Spacecraft and Rockets 52 (3) (2015) 728-738.

[48] C. Xie, G. Zhang, Y. Zhang, Shaping approximation for low-thrust trajectories with large out-of-plane motion, Journal of Guidance, Control, and Dynamics 39 (12) (2016) 2780-2789.

[49] W. E. Wiesel, Modern Astrodynamics, CreateSpace Independent Publishing Platform, 2010.

[50] H. Shang, H. Cui, P. Cui, E. Luan, Optimal-fuel, low-thrust earth-ivar transfer trajectory with venus gravity assist, in: 2006 1st International Symposium on Systems and Control in Aerospace and Astronautics, IEEE, 2006, pp. 1-5.

[51] H. Shang, P. Cui, E. Luan, D. Qiao, Study of design and optimization of low-thrust transfer trajectory with planetary aerogravity assist, in: Computational Engineering in Systems Applications, IMACS Multiconference on, Vol. 1, IEEE, 2006, pp. 690-695.

[52] A. Peloni, C. R. McInnes, M. Ceriotti, Osculating keplerian elements for highly non-keplerian orbits, in: 27th AAS/AIAA Space Flight Mechanics Meeting, 2017, pp. 1-20.

[53] G. R. Hintz, Survey of orbit element sets, Journal of Guidance, Control, and Dynamics 31 (3) (2008) 785-790.

[54] G. R. Hintz, Orbital Mechanics and Astrodynamics, Springer International Publishing, 2015.

[55] J. Li, S. R. Vadali, H. Baoyin, Autonomous lunar orbit rendezvous guidance based on j2-perturbed state transition matrix, Journal of Guidance, Control, and Dynamics 38 (2) (2015) 315-322.

[56] R. Whitley, R. Martinez, Options for staging orbits in cislunar space, in: Aerospace Conference, 2016 IEEE, IEEE, 2016, pp. 1-9.

[57] D. Guzzetti, E. M. Zimovan, K. C. Howell, D. C. Davis, Stationkeeping analysis for spacecraft in lunar near rectilinear halo orbits, in: 27th AAS/AIAA Space Flight Mechanics Meeting, 2017, pp. 1-20. 
[58] A. J. Abraham, D. B. Spencer, T. J. Hart, Early mission design of transfers to halo orbits via particle swarm optimization, The Journal of the Astronautical Sciences 63 (2) (2016) 81-102.

[59] Y. Ulybyshev, Long-term station keeping of space station in lunar halo orbits, Journal of Guidance, Control, and Dynamics 38 (6) (2015) 10631070 .

[60] G. Gomez, M. W. Lo, J. J. Masdemont, Libration Point Orbits and Applications, World Scientific, 2002.

[61] Y. Chen, H. Baoyin, J. Li, Design and optimization of a trajectory for moon departure near earth asteroid exploration, Science China Physics, Mechanics and Astronomy 54 (4) (2011) 748-755.

[62] H. Yang, J. Li, H. Baoyin, Low-cost transfer between asteroids with distant orbits using multiple gravity assists, Advances in Space Research 56 (5) (2015) 837-847.

[63] M. Rasotto, R. Armellin, P. D. Lizia, Multi-step optimization strategy for fuel-optimal orbital transfer of low-thrust spacecraft, Engineering Optimization 48 (3) (2015) 519-542.

[64] K. Zeng, Y. Geng, B. Wu, C. Xie, A novel shape-based approximation method for constrained low-thrust trajectory design, in: AIAA/AAS Astrodynamics Specialist Conference, American Institute of Aeronautics and Astronautics, 2016, pp. 1-9.

[65] E. Taheri, N. I. Li, I. Kolmanovsky, Co-state initialization for the minimum-time low-thrust trajectory optimization, Advances in Space Research 59 (9) (2017) 2360-2373.

[66] J. Roa, J. Peláez, J. Senent, New analytic solution with continuous thrust: Generalized logarithmic spirals, Journal of Guidance, Control, and Dynamics 39 (10) (2016) 2336-2351.

[67] T. Guo, F. Jiang, J. Li, Homotopic approach and pseudospectral method applied jointly to low thrust trajectory optimization, Acta Astronautica 71 (2012) 38-50. 
[68] W. Wang, G. Mengali, A. A. Quarta, J. Yuan, Analysis of relative motion in non-keplerian orbits via modified equinoctial elements, Aerospace Science and Technology 58 (2016) 389 - 400.

[69] I. Shafieenejad, A. Novinzadeh, V. Molazadeh, Comparing and analyzing min-time and min-effort criteria for free true anomaly of low-thrust orbital maneuvers with new optimal control algorithm, Aerospace Science and Technology 35 (2014) 116 - 134.

[70] E. Taheri, I. Kolmanovsky, E. Atkins, Enhanced smoothing technique for indirect optimization of minimum-fuel low-thrust trajectories, Journal of Guidance, Control, and Dynamics 39 (11) (2016) 2500-2511.

[71] M. Vasile, P. D. Pascale, S. Casotto, On the optimality of a shapebased approach based on pseudo-equinoctial elements, Acta Astronautica 61 (1-6) (2007) 286-297.

[72] Y. Lu, H. Li, J. Li, Z. Che, Y. Yang, Y. Yang, Y. Sun, Design and optimization of low-energy transfer orbit to mars with multi-body environment, Science China Technological Sciences 58 (10) (2015) 16601671.

[73] D.-L. Yang, B. Xu, L. Zhang, Optimal low-thrust spiral trajectories using lyapunov-based guidance, Acta Astronautica 126 (2016) 275-285.

[74] Y. Dalin, X. Bo, G. Youtao, Optimal strategy for low-thrust spiral trajectories using lyapunov-based guidance, Advances in Space Research 56 (5) (2015) 865-878.

[75] W. Feng, L. Han, L. Shi, D. Zhao, K. Yang, Optimal control for a cooperative rendezvous between two spacecraft from determined orbits, The Journal of the Astronautical Sciences 63 (1) (2016) 23-46.

[76] G. Avanzini, A. Palmas, E. Vellutini, Solution of low-thrust lambert problem with perturbative expansions of equinoctial elements, Journal of Guidance, Control, and Dynamics 38 (9) (2015) 1585-1601.

[77] X. Yue, Y. Yang, Z. Geng, Indirect optimization for finite-thrust timeoptimal orbital maneuver, Journal of Guidance, Control, and Dynamics 33 (2) (2010) 628-634. 
[78] F. Zuiani, M. Vasile, A. Palmas, G. Avanzini, Direct transcription of low-thrust trajectories with finite trajectory elements, Acta Astronautica 72 (2012) 108-120.

[79] H. Liu, B. H. Tongue, Indirect spacecraft trajectory optimization using modified equinoctial elements, Journal of Guidance, Control, and Dynamics 33 (2) (2010) 619-623.

[80] F. E. Laipert, J. M. Longuski, Low-thrust trajectories for human missions to ceres, Acta Astronautica 95 (2014) 124-132.

[81] X. Yang, X. Cao, A new approach to autonomous rendezvous for spacecraft with limited impulsive thrust: Based on switching control strategy, Aerospace Science and Technology 43 (2015) 454-462.

[82] G. Zhang, D. Ye, Optimal short-range rendezvous using on-off constant thrust, Aerospace Science and Technology 69 (2017) 209-217.

[83] Y. Qi, Y. Jia, Constant thrust fuel-optimal control for spacecraft rendezvous, Advances in Space Research 49 (7) (2012) 1140 - 1150.

[84] L. Ma, X. Meng, Z. Liu, L. Du, Multi-objective and reliable control for trajectory-tracking of rendezvous via parameter-dependent lyapunov functions, Acta Astronautica 81 (1) (2012) 122-136.

[85] G.-J. Tang, Y.-Z. Luo, H.-Y. Li, Optimal robust linearized impulsive rendezvous, Aerospace Science and Technology 11 (7-8) (2007) 563569.

[86] M. Pontani, B. A. Conway, Optimal finite-thrust rendezvous trajectories found via particle swarm algorithm, Journal of Spacecraft and Rockets 50 (6) (2013) 1222-1234.

[87] Y. Ren, J. Shan, A novel algorithm for generating libration point orbits about the collinear points, Celestial Mechanics and Dynamical Astronomy 120 (1) (2014) 57-75.

[88] H. Lei, B. Xu, Y. Sun, Earth-moon low energy trajectory optimization in the real system, Advances in Space Research 51 (5) (2013) 917-929. 
[89] H. Peng, Q. Gao, Z. Wu, W. Zhong, Optimal guidance based on receding horizon control for low-thrust transfer to libration point orbits, Advances in Space Research 51 (11) (2013) 2093-2111.

[90] D. C. Folta, N. Bosanac, D. Guzzetti, K. C. Howell, An earth-moon system trajectory design reference catalog, Acta Astronautica 110 (2015) 341-353.

[91] Y. Chen, H. Baoyin, J. Li, Trajectory design for the moon departure libration point mission in full ephemeris model, Science China Technological Sciences 54 (11) (2011) 2924-2934.

[92] H. Lei, B. Xu, Low-energy transfers to cislunar periodic orbits visiting triangular libration points, Communications in Nonlinear Science and Numerical Simulation 54 (2018) 466-481.

[93] R. Marler, J. Arora, Survey of multi-objective optimization methods for engineering, Structural and Multidisciplinary Optimization 26 (6) (2004) 369-395.

[94] B. Derbel, D. Brockhoff, A. Liefooghe, S. Verel, On the impact of multiobjective scalarizing functions, in: International Conference on Parallel Problem Solving from Nature, Springer, 2014, pp. 548-558.

[95] M. K. Fain, O. L. Starinova, Ballistic optimization of the 11-12 and 12-11 low thrust transfers in the earth-moon system, in: 2015 7th International Conference on Recent Advances in Space Technologies (RAST), 2015, pp. 95-98.

[96] Y. Wang, M. Zhu, Y. Wei, Y. Zhang, Solar sail spacecraft trajectory optimization based on improved imperialist competitive algorithm, in: Intelligent Control and Automation (WCICA), 2012 10th World Congress on, IEEE, 2012, pp. 191-195.

[97] J. Zhao, R. Zhou, Pigeon-inspired optimization applied to constrained gliding trajectories, Nonlinear Dynamics 82 (4) (2015) 1781-1795.

[98] N. Yokoyama, S. Suzuki, Modified genetic algorithm for constrained trajectory optimization, Journal of Guidance, Control, and Dynamics 28 (1) (2005) 139-144. 
[99] G. Huntington, D. Benson, A. Rao, A comparison of accuracy and computational efficiency of three pseudospectral methods, AIAA Guidance, Navigation and Control Conference and Exhibit.

[100] A. V. Ivanyukhin, V. G. Petukhov, The thrust minimization problem and its applications, Cosmic Research 53 (4) (2015) 300-310.

[101] E. Taheri, O. Abdelkhalik, Initial three-dimensional low-thrust trajectory design, Advances in Space Research 57 (3) (2016) 889 - 903.

[102] Y. Ulybyshev, Trajectory optimization for spacecraft proximity operations with constraints, in: AIAA Guidance, Navigation, and Control Conference, American Institute of Aeronautics and Astronautics, 2011, pp. 1-19.

[103] Y. P. Ulybyshev, Optimization of low-thrust orbit transfers with constraints, Cosmic Research 50 (5) (2012) 376-390.

[104] L. W. Neustadt, A general theory of minimum-fuel space trajectories, Journal of the Society for Industrial and Applied Mathematics, Series A: Control 3 (2) (1965) 317-356.

[105] Y.-Z. Luo, G.-J. Tang, Spacecraft optimal rendezvous controller design using simulated annealing, Aerospace Science and Technology 9 (8) (2005) 732-737.

[106] S. Li, R. Mehra, R. Smith, R. Beard, Multi-spacecraft trajectory optimization and control using genetic algorithm techniques, in: 2000 IEEE Aerospace Conference. Proceedings (Cat. No.00TH8484), IEEE, 2000, pp. 99-108.

[107] B. M. Shippey, Trajectory optimization using collocation and evolutionary programming for constrained nonlinear dynamical systems, Ph.D. thesis, The University of Texas at Arlington (2008).

[108] K. Miettinen, M. M. Makela, On scalarizing functions in multiobjective optimization, OR Spectrum 24 (2) (2002) 193-213.

[109] J. W. Sales Jr, Trajectory optimization for spacecraft collision avoidance, Tech. rep., Air Force Institute of Technology (2013). 
[110] Y. Zhou, Y. Yan, X. Huang, L. Kong, Mission planning optimization for multiple geosynchronous satellites refueling, Advances in Space Research 56 (11) (2015) 2612-2625.

[111] Z. Tarzi, J. Speyer, R. Wirz, Fuel optimum low-thrust elliptic transfer using numerical averaging, Acta Astronautica 86 (2013) 95- 118.

[112] M. Pontani, B. A. Conway, Particle swarm optimization applied to space trajectories, Journal of Guidance, Control, and Dynamics 33 (5) (2010) 1429-1441.

[113] A. Bolle, C. Circi, A hybrid, self-adjusting search algorithm for optimal space trajectory design, Advances in Space Research 50 (4) (2012) 471 -488 .

[114] A. Shirazi, A. Mazinan, Mathematical modeling of spacecraft guidance and control system in 3d space orbit transfer mission, Computational and Applied Mathematics 35 (3) (2016) 865-879.

[115] R. Vinter, Optimal control, Springer Science \& Business Media, 2010.

[116] A. A. Quarta, G. Mengali, Semi-analytical method for the analysis of solar sail heliocentric orbit raising, Journal of Guidance, Control, and Dynamics 35 (1) (2012) 330-335.

[117] O. von Stryk, R. Bulirsch, Direct and indirect methods for trajectory optimization, Annals of Operations Research 37 (1) (1992) 357-373.

[118] I. M. Ross, A historical introduction to the convector mapping principle, in: Proceedings of Astrodynamics Specialists Conference, Naval Postgraduate School (US), 2005, pp. 1-21.

[119] I. M. Ross, F. Fahroo, A pseudospectral transformation of the convectors of optimal control systems, IFAC Proceedings Volumes 34 (13) (2001) 543-548.

[120] I. M. Ross, F. Fahroo, A unified computational framework for realtime optimal control, in: Decision and Control, 2003. Proceedings. 42nd IEEE Conference on, Vol. 3, IEEE, 2003, pp. 2210-2215. 
[121] M. Ross, F. Fahroo, Discrete verification of necessary conditions for switched nonlinear optimal control systems, in: American Control Conference, 2004. Proceedings of the 2004, Vol. 2, IEEE, 2004, pp. 16101615 .

[122] F. L. Lewis, D. L. Vrabie, V. L. Syrmos, Optimal Control, John Wiley \& Sons, Inc., 2012.

[123] S. da Silva Fernandes, F. das Chagas Carvalho, R. V. de Moraes, Optimal low-thrust transfers between coplanar orbits with small eccentricities, Computational and Applied Mathematics 35 (3) (2015) 803-816.

[124] A. V. Rao, A survey of numerical methods for optimal control, Advances in the Astronautical Sciences 135 (2009) 497 - 528.

[125] J. Z. Ben-Asher, Optimal Control Theory with Aerospace Applications, American Institute of Aeronautics and Astronautics, 2010.

[126] O. Abdelkhalik, E. Taheri, Shape based approximation of constrained low-thrust space trajectories using fourier series, Journal of Spacecraft and Rockets 49 (3) (2012) 535-546.

[127] D. P. Bertsekas, Dynamic Programming and Optimal Control, 4th Edition, Vol. 2, Athena Scientific, 2016.

[128] A. Bressan, G. Facchi, Trajectories of differential inclusions with state constraints, Journal of Differential Equations 250 (4) (2011) 2267-2281.

[129] M. Diehl, H. G. Bock, H. Diedam, P.-B. Wieber, Fast direct multiple shooting algorithms for optimal robot control, in: Fast motions in biomechanics and robotics, Springer, 2006, pp. 65-93.

[130] D. A. Benson, G. T. Huntington, T. P. Thorvaldsen, A. V. Rao, Direct trajectory optimization and costate estimation via an orthogonal collocation method, Journal of Guidance, Control, and Dynamics 29 (6) (2006) 1435-1440.

[131] A. V. Rao, D. A. Benson, C. Darby, M. A. Patterson, C. Francolin, I. Sanders, G. T. Huntington, Algorithm 902: Gpops, a matlab software for solving multiple-phase optimal control problems using the gauss pseudospectral method, ACM Trans. Math. Softw. 37 (2) (2010) 22:122:39. 
[132] D. Garg, M. A. Patterson, C. Francolin, C. L. Darby, G. T. Huntington, W. W. Hager, A. V. Rao, Direct trajectory optimization and costate estimation of finite-horizon and infinite-horizon optimal control problems using a radau pseudospectral method, Computational Optimization and Applications 49 (2) (2011) 335-358.

[133] H. Hou, W. Hager, A. Rao, Convergence of a gauss pseudospectral method for optimal control, AIAA Guidance, Navigation, and Control Conference.

[134] D. Garg, M. Patterson, W. W. Hager, A. V. Rao, D. A. Benson, G. T. Huntington, A unified framework for the numerical solution of optimal control problems using pseudospectral methods, Automatica 46 (11) (2010) $1843-1851$.

[135] D. Garg, W. W. Hager, A. V. Rao, Pseudospectral methods for solving infinite-horizon optimal control problems, Automatica 47 (4) (2011) 829-837.

[136] D. Garg, M. A. Patterson, W. W. Hager, A. V. Rao, D. Benson, G. T. Huntington, An overview of three pseudospectral methods for the numerical solution of optimal control problems, Advances in the Astronautical Sciences (2009) 475 - 487.

[137] X. Guo, M. Zhu, Direct trajectory optimization based on a mapped chebyshev pseudospectral method, Chinese Journal of Aeronautics 26 (2) (2013) $401-412$.

[138] Q. Gong, F. Fahroo, I. M. Ross, et al., Spectral algorithm for pseudospectral methods in optimal control, Journal of Guidance Control and Dynamics 31 (3) (2008) 460-471.

[139] P. Williams, Hermite-legendre-gauss-lobatto direct transcription in trajectory optimization, Journal of Guidance, Control, and Dynamics 32 (4) (2009) 1392-1395.

[140] I. M. Ross, M. Karpenko, A review of pseudospectral optimal control: From theory to flight, Annual Reviews in Control 36 (2) (2012) 182 197. 
[141] F. Topputo, C. Zhang, Survey of direct transcription for low-thrust space trajectory optimization with applications, Abstract and Applied Analysis 2014 (2014) 1-15.

[142] A. Engelsone, S. L. Campbell, Adjoint estimation using direct transcription multipliers: compressed trapezoidal method, Optimization and Engineering 9 (3) (2008) 291-305.

[143] O. Abdelkhalik, E. Taheri, Approximate on-off low-thrust space trajectories using fourier series, Journal of Spacecraft and Rockets 49 (5) (2012) 962-965.

[144] Y. Ulybyshev, Discrete pseudocontrol sets for optimal control problems, Journal of Guidance, Control, and Dynamics 33 (4) (2010) 11331142 .

[145] S. Tang, B. A. Conway, Optimization of low-thrust interplanetary trajectories using collocation and nonlinear programming, Journal of Guidance, Control, and Dynamics 18 (3) (1995) 599-604.

[146] I. Shafieenejad, A. Novinzadeh, V. Molazadeh, Introducing a novel algorithm for minimum-time low-thrust orbital transfers with free initial condition, Proceedings of the Institution of Mechanical Engineers, Part G: Journal of Aerospace Engineering 229 (2) (2014) 333-351.

[147] S. Zhao, J. Zhang, Minimum-fuel station-change for geostationary satellites using low-thrust considering perturbations, Acta Astronautica 127 (2016) 296-307.

[148] B. N. Kiforenko, I. Y. Vasiliev, Numerical solutions to exact equations of motion along near-optimal multi-orbit trajectories for a spacecraft in a newtonian gravitational field, Cosmic Research 49 (5) (2011) 424439.

[149] C. L. Ranieri, C. A. Ocampo, Indirect optimization of spiral trajectories, Journal of Guidance, Control, and Dynamics 29 (6) (2006) 13601366 .

[150] M. Vavrina, K. Howell, Global low-thrust trajectory optimization through hybridization of a genetic algorithm and a direct method, AIAA/AAS Astrodynamics Specialist Conference and Exhibit. 
[151] E. Taheri, O. Abdelkhalik, Fast initial trajectory design for low-thrust restricted-three-body problems, Journal of Guidance, Control, and Dynamics 38 (11) (2015) 2146-2160.

[152] D. M. Novak, M. Vasile, Improved shaping approach to the preliminary design of low-thrust trajectories, Journal of Guidance, Control, and Dynamics 34 (1) (2011) 128-147.

[153] Z. B. Tarzi, Optimum low thrust elliptic orbit transfer using numerical averaging, University of California, Los Angeles, 2012.

[154] N. Sullo, A. Peloni, M. Ceriotti, Low-thrust to solar-sail trajectories: A homotopic approach, Journal of Guidance, Control, and Dynamics (2017) 1-11.

[155] Y. Ulybyshev, Stationkeeping strategy and possible lunar halo orbits for long-term space station, in: AIAA Guidance, Navigation, and Control Conference, American Institute of Aeronautics and Astronautics, 2014, pp. 1-16.

[156] H. Ma, S. Xu, Global fuel consumption optimization of an open-time terminal rendezvous and docking with large-eccentricity elliptic-orbit by the method of interval analysis, Acta Astronautica 128 (2016) 593606.

[157] H. Peng, X. Jiang, Nonlinear receding horizon guidance for spacecraft formation reconfiguration on libration point orbits using a symplectic numerical method, ISA Transactions 60 (2016) 38-52.

[158] M. T. Ozimek, K. C. Howell, Low-thrust transfers in the earth-moon system, including applications to libration point orbits, Journal of Guidance, Control, and Dynamics 33 (2) (2010) 533-549.

[159] M. Sayanjali, S. H. Pourtakdoust, Optimal trajectory design to halo orbits via pseudo-invariant manifolds using a nonlinear four body formulation, Acta Astronautica 110 (2015) 115-128.

[160] L. S. Breger, J. P. How, Safe trajectories for autonomous rendezvous of spacecraft, Journal of Guidance, Control, and Dynamics 31 (5) (2008) $1478-1489$. 
[161] K. Zeng, Y. Geng, B. Wu, Shape-based analytic safe trajectory design for spacecraft equipped with low-thrust engines, Aerospace Science and Technology 62 (2017) 87-97.

[162] I. S. Grigoriev, M. P. Zapletin, One optimization problem for trajectories of spacecraft rendezvous mission to a group of asteroids, Cosmic Research 47 (5) (2009) 426-437.

[163] M. Pontani, B. Conway, Optimal low-thrust orbital maneuvers via indirect swarming method, Journal of Optimization Theory and Applications 162 (1) (2013) 272-292.

[164] M. Pontani, B. A. Conway, Minimum-fuel finite-thrust relative orbit maneuvers via indirect heuristic method, Journal of Guidance, Control, and Dynamics 38 (5) (2014) 913-924.

[165] Y. Mao, D. Zhang, L. Wang, Reentry trajectory optimization for hypersonic vehicle based on improved gauss pseudospectral method, Soft Computing 21 (16) (2016) 4583-4592.

[166] J. Garcia-Heras, M. Soler, F. J. Saez, A comparison of optimal control methods for minimum fuel cruise at constant altitude and course with fixed arrival time, Procedia Engineering 80 (2014) 231 - 244, 3rd International Symposium on Aircraft Airworthiness (ISAA 2013).

[167] A. Miele, A. Salvetti, Applied Mathematics in Aerospace Science and Engineering, Springer, 2014.

[168] S. Campagnola, P. Skerritt, R. P. Russell, Flybys in the planar, circular, restricted, three-body problem, Celestial Mechanics and Dynamical Astronomy 113 (3) (2012) 343-368.

[169] G. Mengali, A. A. Quarta, Escape from elliptic orbit using constant radial thrust, Journal of Guidance, Control, and Dynamics 32 (3) (2009) $1018-1022$.

[170] P. E. Gill, W. Murray, M. A. Saunders, Snopt: An sqp algorithm for large-scale constrained optimization, SIAM review 47 (1) (2005) 99131. 
[171] P. Spellucci, Donlp2 short users guide, Technische Universitt Darmstadt.

[172] R. Fletcher, S. Leyffer, User manual for filtersqp, Numerical Analysis Report NA/181, Department of Mathematics, University of Dundee, Dundee, Scotland (1998) 35.

[173] R. A. Bartlett, L. T. Biegler, rsqp++: An object-oriented framework for successive quadratic programming, in: Large-Scale PDEConstrained Optimization, Springer, 2003, pp. 316-330.

[174] R. H. Byrd, J. Nocedal, R. A. Waltz, Knitro: An integrated package for nonlinear optimization, in: Large-scale nonlinear optimization, Springer, 2006, pp. 35-59.

[175] W. Wang, H. Peng, A fast multi-objective optimization design method for emergency libration point orbits transfer between the sun-earth and the earth-moon systems, Aerospace Science and Technology 63 (2017) $152-166$.

[176] J. Xiao-yong, Z. Hong-bo, T. Guo-jian, A novel low-thrust trajectory optimization approach based on virtual gravitational body, in: 2013 Chinese Automation Congress, IEEE, 2013, pp. 295-299.

[177] M. Walmsley, J. Heiligers, M. Ceriotti, C. McInnes, Optimal trajectories for planetary pole-sitter missions, Journal of Guidance, Control, and Dynamics 39 (10) (2016) 2461-2468.

[178] P. V. Anderson, H. Schaub, N-impulse formation flying feedback control using nonsingular element description, Journal of Guidance, Control, and Dynamics 37 (2) (2014) 540-548.

[179] D. Pérez, R. Bevilacqua, Differential drag-based reference trajectories for spacecraft relative maneuvering using density forecast, Journal of Spacecraft and Rockets 53 (1) (2016) 234-239.

[180] T. Chen, E. van Kampen, H. Yu, Q. P. Chu, Optimization of timeopen constrained lambert rendezvous using interval analysis, Journal of Guidance, Control, and Dynamics 36 (1) (2013) 175-184. 
[181] Neos server for optimization, (Online; accessed 30-May-2018). URL https://neos-server.org/

[182] Aimms: Prescriptive analytics and supply chain management, (Online; accessed 30-May-2018).

URL https://aimms. com/

[183] Ampl: Streamlined modeling for real optimization, (Online; accessed 30-May-2018).

URL https://ampl.com/

[184] Gams: The general algebraic modeling system, (Online; accessed 30May-2018).

URL https://gams.com/

[185] D. P. Bertsekas, Nonlinear programming, Athena scientific Belmont, 2016.

[186] H. Seywald, R. R. Kumar, S. M. Deshpande, Genetic algorithm approach for optimal control problems with linearly appearing controls, Journal of Guidance, Control, and Dynamics 18 (1) (1995) 177-182.

[187] S. Voß, S. Martello, I. H. Osman, C. Roucairol (Eds.), Meta-Heuristics: Advances and Trends in Local Search Paradigms for Optimization, Springer US, 1998.

[188] C. Blum, A. Roli, Metaheuristics in combinatorial optimization: Overview and conceptual comparison, ACM Comput. Surv. 35 (3) (2003) 268-308.

[189] R. Popa, Genetic Algorithms in Applications, InTech, 2012.

[190] O. Kramer, Machine Learning for Evolution Strategies, Springer International Publishing, 2016.

[191] R. Riolo, E. Vladislavleva, M. D. Ritchie, J. H. Moore, Genetic Programming Theory and Practice X, Springer, 2013.

[192] E. P. Konstantinos, N. V. Michael, Introduction, in: Particle Swarm Optimization and Intelligence, IGI Global, 2010, pp. 1-24. 
[193] N. Xiong, D. Molina, M. L. Ortiz, F. Herrera, A walk into metaheuristics for engineering optimization: Principles, methods and recent trends, International Journal of Computational Intelligence Systems 8 (4) (2015) 606-636.

[194] M. A. Muñoz, Y. Sun, M. Kirley, S. K. Halgamuge, Algorithm selection for black-box continuous optimization problems: A survey on methods and challenges, Information Sciences 317 (2015) $224-245$.

[195] I. F. Jr., X. Yang, I. Fister, J. Brest, D. Fister, A brief review of nature-inspired algorithms for optimization, CoRR abs/1307.4186.

[196] M. R. Sentinella, L. Casalino, Cooperative evolutionary algorithm for space trajectory optimization, Celestial Mechanics and Dynamical Astronomy 105 (1-3) (2009) 211-227.

[197] Y.-Z. Luo, G.-J. Tang, Rendezvous phasing special-point maneuvers mixed discrete-continuous optimization using simulated annealing, Aerospace Science and Technology 10 (7) (2006) 652-658.

[198] M. Pontani, P. Ghosh, B. A. Conway, Particle swarm optimization of multiple-burn rendezvous trajectories, Journal of Guidance, Control and Dynamics 35 (4) (2012) 1192-1207.

[199] M. Pontani, B. A. Conway, Particle swarm optimization applied to impulsive orbital transfers, Acta Astronautica 74 (2012) 141-155.

[200] B. A. Rogers, K. M. Hughes, J. M. Longuski, B. Aldrin, Establishing cycler trajectories between earth and mars, Acta Astronautica 112 (2015) $114-125$.

[201] P. Cage, I. Kroo, R. Braun, Interplanetary trajectory optimization using a genetic algorithm, in: Astrodynamics Conference, American Institute of Aeronautics and Astronautics, 1994, pp. 538-547.

[202] F. A. Zotes, M. S. Peñas, Particle swarm optimisation of interplanetary trajectories from earth to jupiter and saturn, Engineering Applications of Artificial Intelligence 25 (1) (2012) 189-199.

[203] K. Zhu, J. Li, H. Baoyin, Multi-swingby optimization of mission to saturn using global optimization algorithms, Acta Mechanica Sinica 25 (6) (2009) 839-845. 
[204] B. Carpenter, B. Jackson, Stochastic optimization of spacecraft rendezvous trajectories, in: 2003 IEEE Aerospace Conference Proceedings (Cat. No.03TH8652), Vol. 8, IEEE, 2003, pp. 3731-3738.

[205] B. B. K. Reddy, A. Homaifar, A. C. Esterline, Thrust control of an electric propulsion space vehicle with minimal fuel consumption, Intelligent Automation \& Soft Computing 14 (3) (2008) 333-349.

[206] J. A. Englander, B. A. Conway, Automated solution of the low-thrust interplanetary trajectory problem, Journal of Guidance, Control, and Dynamics 40 (1) (2017) 15-27.

[207] B. Wall, B. A. Conway, Near-optimal low-thrust earth-mars trajectories via a genetic algorithm, Journal of Guidance, Control, and Dynamics 28 (5) (2005) 1027-1031.

[208] J. Shan, Y. Ren, Low-thrust trajectory design with constrained particle swarm optimization, Aerospace Science and Technology 36 (2014) 114124.

[209] M. Ceriotti, J. P. Sanchez, Control of asteroid retrieval trajectories to libration point orbits, Acta Astronautica 126 (2016) 342 - 353, space Flight Safety.

[210] N. Assadian, S. H. Pourtakdoust, Multiobjective genetic optimization of earth-moon trajectories in the restricted four-body problem, Advances in Space Research 45 (3) (2010) 398-409.

[211] X. Pan, M. Xu, R. Santos, Trajectory optimization for solar sail in cislunar navigation constellation with minimal lightness number, Aerospace Science and Technology.

[212] Y.-Z. Luo, G.-J. Tang, L.-N. Zhou, Simulated annealing for solving near-optimal low-thrust orbit transfer, Engineering Optimization 37 (2) (2005) 201-216.

[213] A. Shirazi, Multi-objective optimization of orbit transfer trajectory using imperialist competitive algorithm, in: 2017 IEEE Aerospace Conference, 2017, pp. 1-14. 
[214] A. Shirazi, J. Ceberio, J. A. Lozano, Evolutionary algorithms to optimize low-thrust trajectory design in spacecraft orbital precession mission, in: 2017 IEEE Congress on Evolutionary Computation (CEC), IEEE, 2017, pp. 1779-1786.

[215] B. Dachwald, Optimization of very-low-thrust trajectories using evolutionary neurocontrol, Acta Astronautica 57 (2-8) (2005) 175-185.

[216] H.-D. Kim, O.-C. Jung, H. Bang, A computational approach to reduce the revisit time using a genetic algorithm, in: 2007 International Conference on Control, Automation and Systems, 2007, pp. 184-189.

[217] A. Shirazi, Analysis of a hybrid genetic simulated annealing strategy applied in multi-objective optimization of orbital maneuvers, IEEE Aerospace and Electronic Systems Magazine 32 (1) (2017) 6-22.

[218] A. Shirazi, Trajectory optimization of spacecraft high-thrust orbit transfer using a modified evolutionary algorithm, Engineering Optimization 48 (10) (2016) 1639-1657. 